\title{
On the simulation of thick non-neutral boundary layers for urban studies in a wind tunnel
}

\author{
D. Marucci ${ }^{\mathrm{a}, *}$, M. Carpentieri ${ }^{\mathrm{a}}$, P. Hayden ${ }^{\mathrm{a}}$ \\ ${ }^{a}$ EnFlo Laboratory, Department of Mechanical Engineering Sciences, University of Surrey, \\ Guildford, Surrey GU2 7XH, UK
}

\begin{abstract}
Stable and convective boundary layers over a very rough surface have been studied in a thermally-stratified wind tunnel. Artificial thickening by means of spires was used to accelerate the formation of a sufficiently deep boundary layer, suitable for urban-like boundary layer flow and dispersion studies. For the stable boundary layer, the methodology presented in Hancock and Hayden (2018) for low-roughness offshore surface conditions has been successfully applied to cases with higher-roughness. Different levels of stratification and roughness produced modifications in the turbulence profiles of the lower half of the boundary layer, but little or no change in the region above. Data for a stronger stability case suggested that the employed spires may not be suitable to simulate such extreme condition, though further studies are needed. The results were in reasonably good agreement with field measurements. For the convective boundary layer, great attention was given to the flow uniformity inside the test section. The selection of a non-uniform inlet temperature profile was in this case found not as determinant as for the stable boundary layer to improve the longitudinal uniformity, while the application of a calibrated capping inversion considerably improved the lateral uniformity. The non-dimensional vertical profiles of turbulent quantities and heat fluxes, did not seem to be influenced by roughness.
\end{abstract}

Keywords: Stable boundary layer, Convective boundary layer, Urban

\footnotetext{
${ }^{*}$ Corresponding author

Email address: d.marucci@surrey.ac.uk (D. Marucci)
} 
boundary layer, Wind tunnel experiments

\section{Introduction}

Atmospheric stratification is due to variations in temperature and humidity with height. A near-adiabatic profile of potential temperature is present in a neutrally stratified atmosphere, where vertical motions of fluid particles are neither amplified nor damped, while an unstable (or convective) stratification is characterised by an enhancement of vertical movements and stable flows are characterised by attenuated vertical motion. Stability affects the atmospheric boundary layer (ABL) depth and structure as well as velocity, temperature and turbulence profiles within it.

Non-neutral stratified conditions are frequently found in atmospheric flows. The data analysis by Argyle and Watson (2012) indicated that non-neutral conditions were present for $70 \%$ of the time in two UK offshore wind farm sites. In urban areas, a large predominance of non-neutral atmosphere was documented, for example, by Wood et al. (2010) over the city of London, UK, with convective cases happening three times more frequently than stable. Nevertheless, most of the experimental and numerical studies focus on neutral flows due to the difficulties in studying atmospheric stratification.

Some experimental studies involving stratified boundary layers (BLs) have been reported so far. The facilities used for this purpose range from water tanks (e.g., Willis and Deardorff, 1974, who simulated a convective boundary layer, CBL, by heating the water from the bottom), saline tanks (like the one in Hibberd and Sawford, 1994, in which the stratification was generated by differences in the salinity level instead of temperature) to wind tunnels. As pointed out by Fedorovich (2004), the first two techniques "omit or treat rather indirectly the effects of wind shears on the turbulence regime", effect that may acquire even more importance when dealing with very rough surfaces, such as urban environments. Thermally-stratified wind tunnels specifically designed for the simulation of stable (SBL) and convective (CBL) boundary layers have 
been built in the past decades. See Meroney (1998) for a review of the main requirements for CBL simulation.

One approach to simulating non-neutral flows in a wind tunnel relies on the development of the BL by means of floor cooling or heating, without any mechanical thickening device. Despite being conceptually simple, such a method requires a very long development region for thick BLs. For example, Arya and Plate (1969) managed to get a $70 \mathrm{~cm}$ thick SBL after $24 \mathrm{~m}$ of growing over a cooled aluminium plate, while Ogawa et al. (1985) obtained a depth of only about $20 \mathrm{~cm}$ after $12 \mathrm{~m}$ on a wind tunnel of similar length. Devices used to artificially thicken the BL include bidimensional blocks or fences, but methods like the ones described by Counihan (1969) and Irwin (1981) for neutral boundary layers (NBLs) give a better control of the BL thickness and turbulence characteristics. The first consists of a castellated barrier wall coupled with a set of quarter-elliptic vorticity generators, while the second makes use of triangular spires, easier to manufacture. Both are normally associated with roughness elements over the floor chosen to provide an aerodynamic rough surface with the desired flow characteristics. Despite being normal practice in NBL simulations, these methods have been rarely used for non-neutral BLs. Robins et al. (2001) employed Counihan's method for the generation of a thick SBL while Hancock et al. (2013), Hancock and Pascheke (2014), Hancock and Hayden (2016) and Hancock and Hayden (2018) used Irwin's spires to develop CBLs and SBLs suitable for low-roughness offshore BL conditions. In the latter two papers, in particular, a method to simulate artificially thickened SBLs was deployed, at least for weak to moderate stability levels and no overlying inversion.

a The effect of roughness in a SBL was studed in the wind tunnel by Williams et al. (2017) and Ohya (2001), who compared his results with the smooth surface simulations by Ohya et al. (1997). Both concluded that turbulence characteristics remain substantially similar, with small differences attributed by the former to changes in local stratification. In their work different levels of stability were considered, ranging from weak to very stable. The transition between weak and strong stability conditions were found by Ohya (Ohya et al., 1997; Ohya, 2001) 
to happen at a value of the bulk Richardson number equal to 0.25 for both smooth and rough surfaces, while Williams et al. (2017) reported two different values, both of them lower than Ohya's results $(0.10$ for the smooth surface and 0.15 for the rough one) with turbulence stress scaling with wall shear only before the transition.

口 As far as CBL simulations are concerned, Fedorovich et al. (1996) and Fedorovich and Kaiser (1998) carried out wind tunnel experiments, finding roughness and wind shear to be responsible of modifications in the regime of turbulence production, with an increment of the velocity variances closer to the surface respect to the shear-free case for values of the surface shear-to-buoyancy production ratio $u_{*} / w_{*}$ greater than 0.3 .

The present work aims to investigate techniques for the development of thick high-roughness SBLs and CBLs suitable for studying flow and dispersion in urban areas. For this purpose the method presented by Hancock and Hayden (2018) for SBLs has been applied with success to a higher roughness case. Artificially thickened CBLs have also been investigated. In this case great efforts were put on the enhancement of longitudinal and lateral uniformity of the temperature and velocity fields. In particular, the use of different inlet temperature gradients as well as an overlying inversion have been tested for this purpose.

\section{Methodology}

\subsection{Experimental setup}

Flow measurements were performed in the suck-down open-return EnFlo meteorological wind tunnel with a test section $20 \mathrm{~m}$ long, $3.5 \mathrm{~m}$ wide and $1.5 \mathrm{~m}$ high. The $x$-axis was in the streamwise direction, measured from the workingsection inlet; the $y$-axis was in the spanwise direction, measured from the wind tunnel centre line; the $z$-axis represented the vertical, starting from the floor. The wind tunnel flow speed could range from 0.3 to $2.5 \mathrm{~m} / \mathrm{s}$ as measured by a sonic anemometer placed at $x=5 \mathrm{~m}, y=1 \mathrm{~m}, z=1 \mathrm{~m}$ (which provided a reference velocity $\left.U_{\mathrm{REF}}\right)$. 
The wind tunnel was specifically designed to generate thermally stratified flows: a series of 15 vertically piled electrical heaters at the inlet section allowed the generation of a vertical temperature gradient, which combined with the heating/cooling floor system created the different types of atmospheric stability. For stable stratification the central $3 \mathrm{~m}$ of the floor along most of the working section in the streamwise direction were cooled by means of recirculating water at the desired temperature. When CBLs had to be simulated, electrical heater mats were added on the wind tunnel floor (on top of additional insulating panels). Their maximum power was $2.0 \mathrm{~kW} / \mathrm{m}^{2}$, with dimensions $1295 \times 333 \times 5 \mathrm{~mm}$; different arrangements were considered in order to improve the lateral uniformity (further explanation will be given in the following sections). Panel temperatures were controlled in a closed-loop system. The air leaving the wind tunnel was cooled by means of recirculating water in order to keep the laboratory temperature as constant as possible. The latter presented a vertical variation up to $1^{\circ} \mathrm{C}$ between floor and ceiling. Such a gradient was mitigated using a series of fans that helped air mixing, improving the temperature homogeneity at the inlet.

As the main purpose of the work is the development of stratified BLs suitable for urban studies (for which the micro-scale was of interest), a wind-tunnel scale of 1:200 was considered for all the cases. In order to obtain sufficiently thick BLs, Irwin-like spires (Irwin, 1981) after the inlet section and rectangular-shaped roughness elements on the floor were employed to artificially develop the flow. For the CBL simulation five spires $1260 \mathrm{~mm}$ high, $170 \mathrm{~mm}$ wide at the base and spaced laterally $630 \mathrm{~mm}$ were used (shown in Fig. 1). They had been extensively employed in previous works for generating urban neutrally stratified BLs about $1 \mathrm{~m}$ thick $(\delta)$, together with surface roughness elements $80 \mathrm{~mm}$ wide, $20 \mathrm{~mm}$ high and $2 \mathrm{~mm}$ thick placed on the floor in a staggered arrangement with both streamwise and lateral pitches of $240 \mathrm{~mm}$. For the stable stratification case a shallower BL was required for scaling issues: in the real atmosphere a SBL tends to be shallower than a NBL or a CBL. Moreover, a shallower BL would allow us to move the measurement traverse to locations outside the BL (the measuring traverse system is currently limited to a maximum height of about $1 \mathrm{~m}$ ). For this 

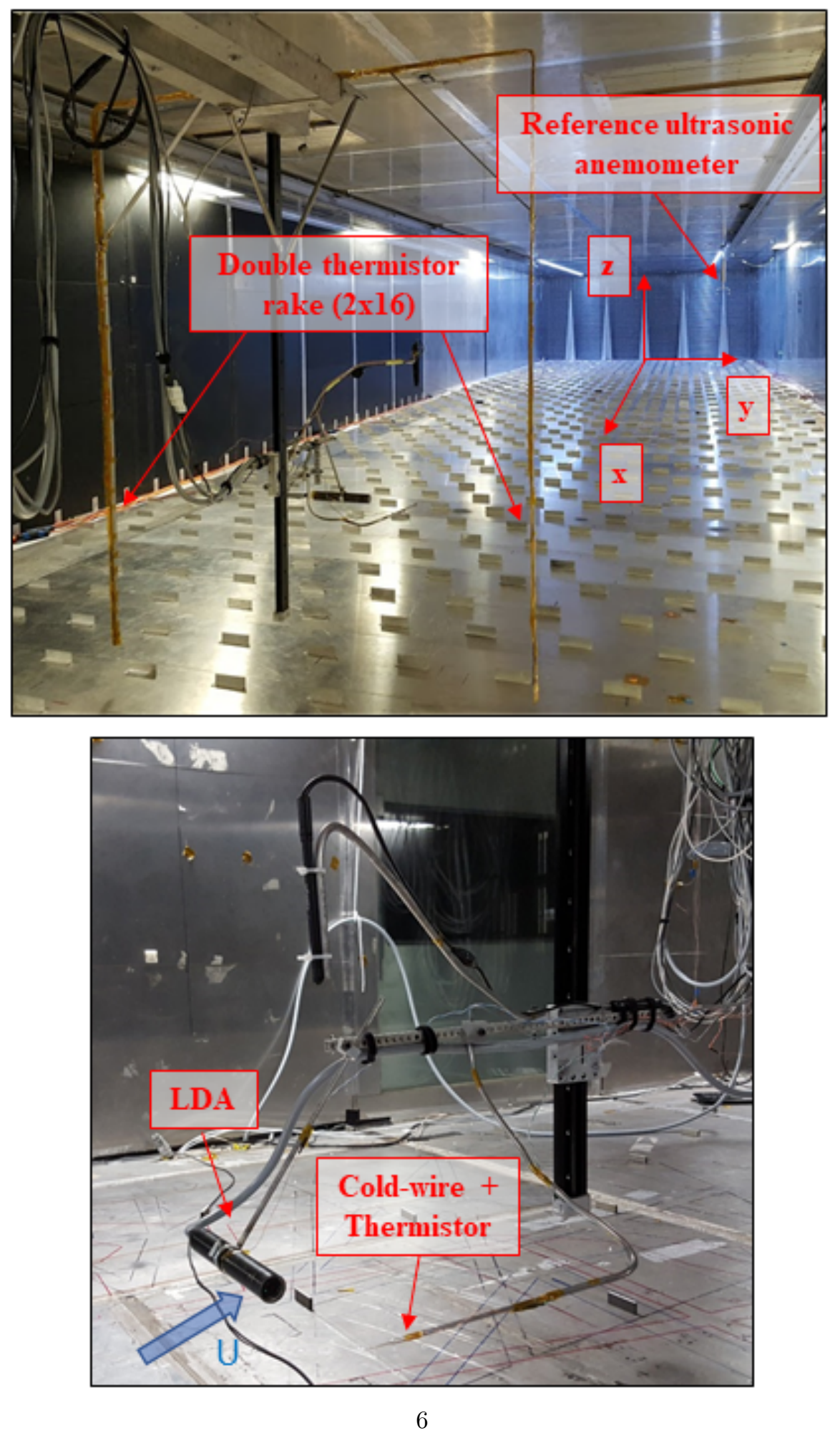

Figure 1: Wind tunnel and measuring setup. 
purpose seven spires $986 \mathrm{~mm}$ high, $121 \mathrm{~mm}$ wide at the base, and $4 \mathrm{~mm}$ wide at the top, spaced laterally $500 \mathrm{~mm}$, were designed according to Irwin's procedure (Irwin, 1981). For all the investigated cases the same roughness element type was employed.

Mean and fluctuating velocity measurements were performed by a twocomponent laser-Doppler anemometer (LDA), via a Dantec $27 \mathrm{~mm}$ FibreFlow probe. For the fluctuating temperature measurements a calibrated fast-response cold-wire probe was used. It was placed about $4 \mathrm{~mm}$ downstream the LDA measuring volume to calculate heat fluxes. This value was chosen in order to reduce the blockage effect of the cold-wire on the measured flow velocity without significantly affecting the correlation between velocity and temperature (see Hancock and Hayden, 2018, Heist and Castro, 1998). Indeed, for a mean convection speed of $0.4 \mathrm{~m} / \mathrm{s}$ (the lowest measured in the dataset) such separation would correspond to a frequency still comparable with the LDA sampling rate. A thermistor was held about $10 \mathrm{~mm}$ on the side of the cold-wire to both measure the mean temperature and calibrate the cold-wire itself. The probes were held by a traverse system mounted on rails on the wind tunnel ceiling, which allowed full three-dimensional movements, ranging from about 6 to $16 \mathrm{~m}$ along $x,-1$ to $1 \mathrm{~m}$ on $y$ and from 0.05 to $1.0 \mathrm{~m}$ on $z$. In order to measure mean temperatures above such a height during the CBL simulation, a second thermistor was placed $430 \mathrm{~mm}$ above the LDA measuring volume. Moreover, a double thermistor rake made up of two series of 16 sensors each was employed in order to acquire the temperature field in the section $(600 \mathrm{~mm}$ downstream the LDA measuring volume). It spanned from $50 \mathrm{~mm}$ to $1350 \mathrm{~mm}$ of height and its acquisition rate was $0.5 \mathrm{~Hz}$. The sampling rate target for the LDA was set to be around $100 \mathrm{~Hz}$, $1000 \mathrm{~Hz}$ for the cold-wire, but with a low-pass filter at $250 \mathrm{~Hz}$. The sampling time for the measurements was 3 minutes both in the SBL and NBL tests, while it was increased up to 5 minutes for the CBL. In the latter, an even longer period was advised based on the scatter between sets of profiles, but this would have increased the experiments duration too much. Data acquisition was performed with the standard LabView based software system of the laboratory. 


\subsection{Measurement scaling}

According to the Monin-Obukhov theory (Monin and Obukhov, 1954), the ratio

$$
\zeta=\frac{z}{L}=-\frac{\left(g / \Theta_{0}\right) \theta_{*}}{u_{*}^{2} / k z}
$$

is the most appropriate stability parameter for the surface layer (SL), defined as the region in which the fluxes varies less the $10 \%$ of their magnitude. The Monin-Obukhov length $L$ is expressed in terms of the friction velocity $u_{*}=$ $\sqrt{-(\overline{u w})_{0}}$ and scaling temperature $\theta_{*}=-(\overline{w \theta})_{0} / u_{*}$, in which $(\overline{w \theta})_{0}$ is the surface kinematic vertical heat flux, $g$ is the acceleration of gravity, $k$ is the von Karman constant (assumed here equal to 0.40) and $\Theta_{0}$ is a reference temperature (here considered as the mean temperature at approximately the aerodynamic roughness length $z_{0}$ height). The wind shear and temperature gradient in the surface layer (which are only function of $\zeta$ ) can then be represented in nondimensional form as

$$
\phi_{m}(\zeta)=\frac{k z}{u_{*}} \frac{\partial U}{\partial z}, \quad \phi_{h}(\zeta)=\frac{k z}{\theta_{*}} \frac{\partial \Theta}{\partial z}
$$

in which $\Theta$ is the mean potential temperature. Integrating them between $z_{0}$ and $z$ leads to the well-known logarithmic expression for the mean streamwise velocity and temperature in the surface layer for the general diabatic case

$$
\begin{gathered}
U(z)=\frac{u_{*}}{k}\left[\ln \left(\frac{z^{\prime}}{z_{0}}\right)-\psi_{m}(\zeta)\right] \\
\Theta(z)-\Theta_{0}=\frac{\theta_{*}}{k}\left[\ln \left(\frac{z^{\prime}}{z_{0 h}}\right)-\psi_{h}(\zeta)\right]
\end{gathered}
$$

where $z^{\prime}=z-d$ to include the contribution of the displacement height $d$, while $z_{0 h}$ is the thermal roughness length. For NBL $\psi_{m}=0$, while for the diabatic case

$$
\psi_{m, h}(\zeta)=\int_{z_{0}, z_{0 h} / L}^{z^{\prime} / L}\left[1-\phi_{m, h}(\zeta)\right] \frac{d \zeta}{\zeta}
$$


In the present work, the parametric equations for $\phi_{m}$ and $\phi_{h}$ for SBL and CBL are determined by fitting with the experimental profiles for the two nondimensional gradients. A reasonable fitting is guaranteed in all the investigated stability cases by the following relations (Eqs. 6 for the SBL and 7 for CBL):

$$
\begin{gathered}
\phi_{m}=1+8 \zeta, \quad \phi_{h}=1+16 \zeta \\
\phi_{m}=(1-16 \zeta)^{-1 / 4}, \quad \phi_{h}=(1-16 \zeta)^{-1 / 2}
\end{gathered}
$$

which are coherent with the forms obtained from field data and summarised in Högström (1988). Forms similar to Eqs. 6 have been found to provide a good fitting also in Hancock and Hayden (2018). Substituted into Eqs. 3 and 4 they lead to the following relations for SBL

$$
\begin{gathered}
U(z)=\frac{u_{*}}{k}\left[\ln \left(z^{\prime} / z_{0}\right)+8 \frac{z^{\prime}-z_{0}}{L}\right] \\
\Theta(z)-\Theta_{0}=\frac{\theta_{*}}{k}\left[\ln \left(\frac{z^{\prime}}{z_{0 h}}\right)+16 \frac{z^{\prime}-z_{0 h}}{L}\right]
\end{gathered}
$$

and for the CBL

$$
\begin{aligned}
U(z)=\frac{u_{*}}{k}\left[\ln \left(\frac{z^{\prime}}{z_{0}}\right)-\ln \left(\frac{(1+\alpha)^{2}}{\left(1+\alpha_{0}\right)^{2}} \frac{\left(1+\alpha^{2}\right)}{\left(1+\alpha_{0}^{2}\right)}\right)\right] \\
+2 \frac{u_{*}}{k}\left(\tan ^{-1}(\alpha)-\tan ^{-1}\left(\alpha_{0}\right)\right)
\end{aligned}
$$

with $\alpha=\left(1-16 z^{\prime} / L\right)^{1 / 4}$ and $\alpha_{0}=\left(1-16 z_{0} / L\right)^{1 / 4}$;

$$
\Theta(z)-\Theta_{0}=\frac{\theta_{*}}{k}\left[\ln \left(\frac{\beta-1}{\beta+1}\right)-\ln \left(\frac{\beta_{0}-1}{\beta_{0}+1}\right)\right]
$$

with $\beta=\left(1-16 z^{\prime} / L\right)^{1 / 2}$ and $\beta_{0}=\left(1-16 z_{0 h} / L\right)^{1 / 2}$. 
The Monin-Obukhov theory also links the value of the gradient Richardson number $\left(R i=g(\partial \Theta / \partial z) /\left[\Theta_{0}(\partial U / \partial z)^{2}\right]\right)$ to the scaling ratio $\zeta$ using

$$
\operatorname{Ri}(\zeta)=\zeta\left(\phi_{h} / \phi_{m}^{2}\right)
$$

While the bulk Richardson number $R i_{b}$ is here calculated as

$$
R i_{b}=\frac{g\left(\Theta_{\delta}-\Theta_{0}\right) \delta}{\Theta_{0} U_{\delta}^{2}}
$$

where $\Theta_{\delta}$ and $U_{\delta}$ are, respectively, the mean temperature and mean streamwise velocity measured at the BL top $\delta$. About the determination of $\delta$ from measured data in the SBL, Stull (1988) listed a series of criteria which might be used, highlighting that a unique method still does not exist and different procedures bring to arbitrary different results. For this reason, in the present study $\delta$ was determine by eye taking into account all the profiles. In particular, $\overline{u w}$ and $\overline{w \theta}$ had to be less than $5 \%$ the values at the surface and $U$ had to be at (or very close to) its free stream value. Differently, for the CBL $\delta$ is usually determine as the height at which $\overline{w \theta}$ experiences a minimum (Stull, 1988). However, in the present study such condition appeared to happen always above the measuring range of the traverse, hence a different method was employed. It considered a fitting of the $\overline{w^{2}}$ profile with a reference curve (as better detailed in Section 4.2 .

For the mixed-layer (ML) of the CBL, differently from the SL, the length scale is considered to be the BL depth $\delta$, while the velocity and temperature scales are, respectively

$$
w_{*}=\left[\frac{g}{\Theta_{0}}(\overline{w \theta})_{0} \delta\right]^{1 / 3}, \quad \tilde{\theta}=\frac{(\overline{w \theta})_{0}}{w_{*}}
$$

as summarised by, e.g., Kaimal and Finnigan (1994).

\subsection{Estimation of surface properties}

In order to estimate $L$, the values of $\overline{u w}$ and $\overline{w \theta}$ at the surface have to be extrapolated from the values in the SL. The same can be said for the profiles of mean streamwise velocity and temperature, used to estimate the roughness 
lengths. However, in an urban boundary layer due to the high level of roughness typically a roughness sub-layer (RSL) develops, in which the measured quantities are not independent from the position relative to local obstacles (here represented by the rectangular roughness elements). The same surface elements shape and arrangement employed here was investigated (among others) by Cheng and Castro (2002) in neutral stratification, for which they found a RSL height equal to $5 H$ (where $H$ is the height of the roughness element). Above such layer they identified an inertial sub-layer (ISL), defined after Oke (1987) as the region where the vertical variation of shear stress was less than $10 \%$ (also called constant flux layer), that extended up to $10 H$. In their work two methods were investigated to estimate $u_{*}$ and $z_{0}$ : the first attempted to use only the points in the ISL where the measured values are expected to be independent from the position, while the second method included also the points in the RSL, but spatially averaged among different locations. In order to evaluate the best solution, both the methods were verified here for SBL, NBL and CBL; the results are shown in Fig. 2 for a location about $12 \mathrm{~m}$ from the inlet. The black points are the values obtained scanning the region downstream a roughness element with a grid of 20 measuring locations (as shown in the map at the bottom of the figure), repeated for 4 heights $(2.5 \mathrm{H}, 5 \mathrm{H}, 7.5 \mathrm{H}, 10 \mathrm{H})$.

For the SBL (a-d in the figure) the data acquired at $2.5 H$ shows a clear dependence from the roughness, effect which is widely reduced at $5 H$, suggesting a similar height for the RSL. However, a constant-flux layer is not clearly identifiable in the region above (the same can be said for the NBL). On this aspect, Cheng et al. (2007) pointed out that a genuine constant-flux layer may not be expected in a wind tunnel with a non-zero pressure gradient as it is the one used here. Despite this, the mean values of $\overline{u w}$ and $\overline{w \theta}$ for each height (red circles in Fig. 2f and d), that are representative of the spatial averaged profile, show a fairly linear trend above $5 H$, which extends down to the floor. This result suggests that a linear fitting in the region above the RSL may be in this case a suitable method to estimate the shear stress and heat flux values at the surface. Such result is confirmed by the analysis of the vertical profiles, one of 
which is reported in the figure (blue squares), which also shows that the linearity extends considerably above $10 H$. For the mean velocity and temperature the variability in the RSL is lower, and both the methods brought generally to a similar solution, having the care to not consider the lower points in case they are too far from the trend of the above region. Similar results were obtained for the NBL (not shown) but with more variability due to the larger turbulence.

For the CBL the points present a higher level of scatter, clearly shown by the two repetitions of the same vertical profile in Fig. 2e-h. The scatter of the measurement makes less evident the local effect of the roughness. Nevertheless, the shear stress profile exhibits an approximately constant flux region which extends from the bottom up to about $25 \mathrm{H}$. A similar trend is shown by the heat flux, but in this case accompanied by a slight reduction below $5 H$. Despite such difference, from the analysis of all the vertical profiles, the most robust method to estimate both $(\overline{u w})_{0}$ and $(\overline{w \theta})_{0}$ appeared to be including all the points of the constant flux region. Finally, for the mean temperature and velocity profiles in the CBL, similar considerations to those for the SBL can be made.

In the following, all the presented parameters are calculated from the average of at least three vertical profiles measured at the centre line and at a distance from the inlet of $12.5,14$ and $15.5 \mathrm{~m}$, in order to have a streamwise average in the region were the boundary layer is reasonably more developed. For part of the CBL data, each profile was also repeated twice to reduce the scatter (only the average of the two repetitions will be shown in the following graphs).

\section{Stable boundary layer}

In this section we firstly discuss about the effects of the main temperature control parameters. Then, we investigate the repeatability of the same stratification at different Reynolds numbers. Finally, we analyse SBLs with moderate stability in more details by comparing the results with data obtained in a NBL, with lower roughness and in field measurements. 

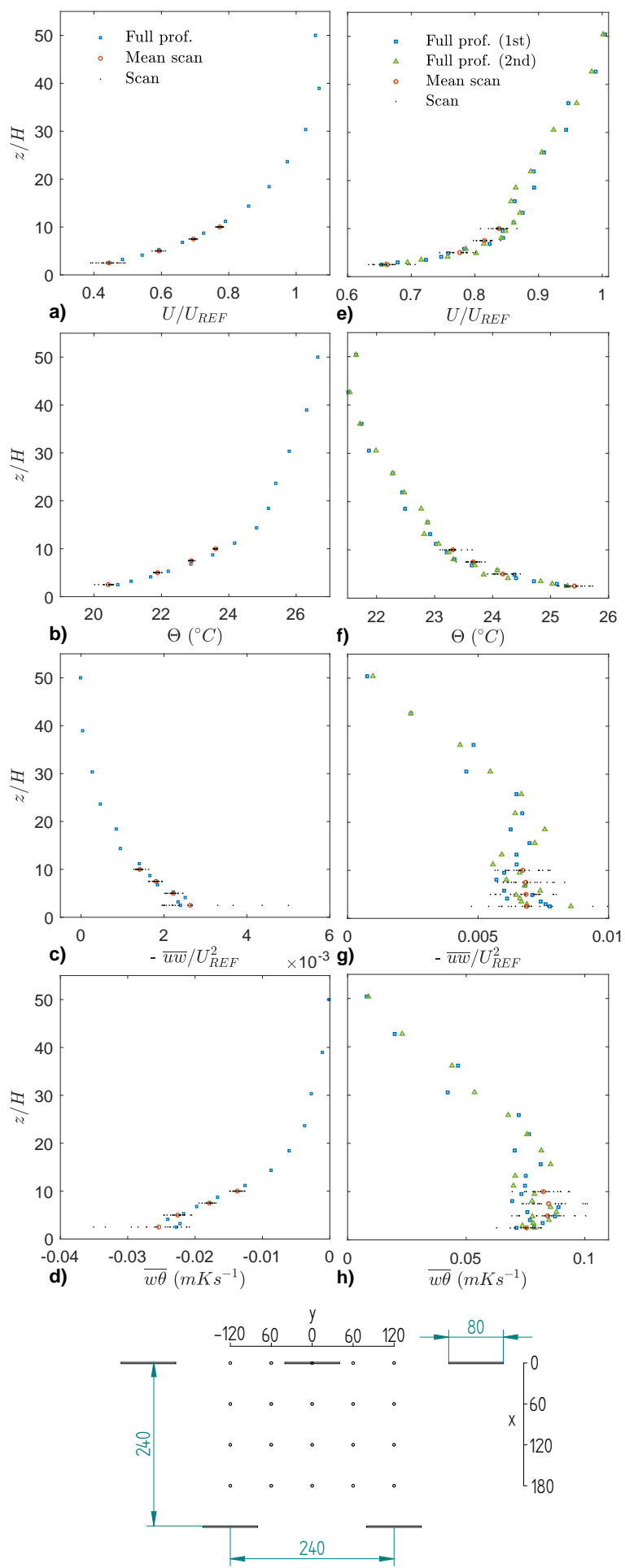

Figure 2: Roughness element scan for $R i_{b}=1 B .14$ (a-d) and -0.5 (e-h). The scan positions are reported in the scheme at the bottom. 


\subsection{Temperature controls}

The three main parameters to be set in order to simulate a SBL in the EnFlo wind tunnel are the maximum temperature difference $\Delta \Theta_{\text {MAX }}$ between cooled floor $\Theta_{0}$ and free stream flow $\Theta_{\infty}$, the length of uncooled floor between the inlet and cooled part, and the imposed temperature profile at the inlet section up to the BL height. A fourth temperature parameter would be the gradient of temperature imposed above the BL, if an overlying inversion were considered. However only zero-strength overlying inversion cases were analysed here in order to reduce the number of parameters.

In the present study, different values for $\Delta \Theta_{\mathrm{MAX}}$ were employed, ranging from 6 to $16^{\circ} \mathrm{C}$. The desired $R i_{b}$ was also obtained modifying the flow velocity and so allowing the air to be cooled by the floor for a different amount of time.

The second parameter to be considered is the length of uncooled floor after the inlet. Previous studies conducted in the EnFlo laboratory for offshore BLs (Hancock and Hayden, 2018) found a dependency of the shear stress $\overline{u w}$ profile from this parameter. Investigating different uncooled floor lengths, the best result in terms of longitudinal uniformity among different locations was found with $5 \mathrm{~m}$ for both the offshore BL and the high-roughness case presented here. More in general, the length of uncooled floor has to be chosen accordingly to the inlet temperature profile.

Finally, a proper inlet temperature gradient has to be considered. The easiest solution would be to impose a uniform inlet temperature and allow the stability to grow thanks to the cooling effect of the floor. However, Hancock and Hayden (2018) found that with this configuration the upper part of the layer remained unaffected by the non-neutral stratification, with a mean temperature constant with height, while temperature fluctuation and heat fluxes approached zero at lower heights than the Reynolds shear stress. This behaviour was likely caused by the advection downstream of the uniform temperature at the inlet (increased by the reduced level of turbulence). On the other hand, also a near constant inlet temperature gradient does not seem to be the best choice. This option was investigated by Ohya and Uchida (2003) and Hancock and Pascheke 


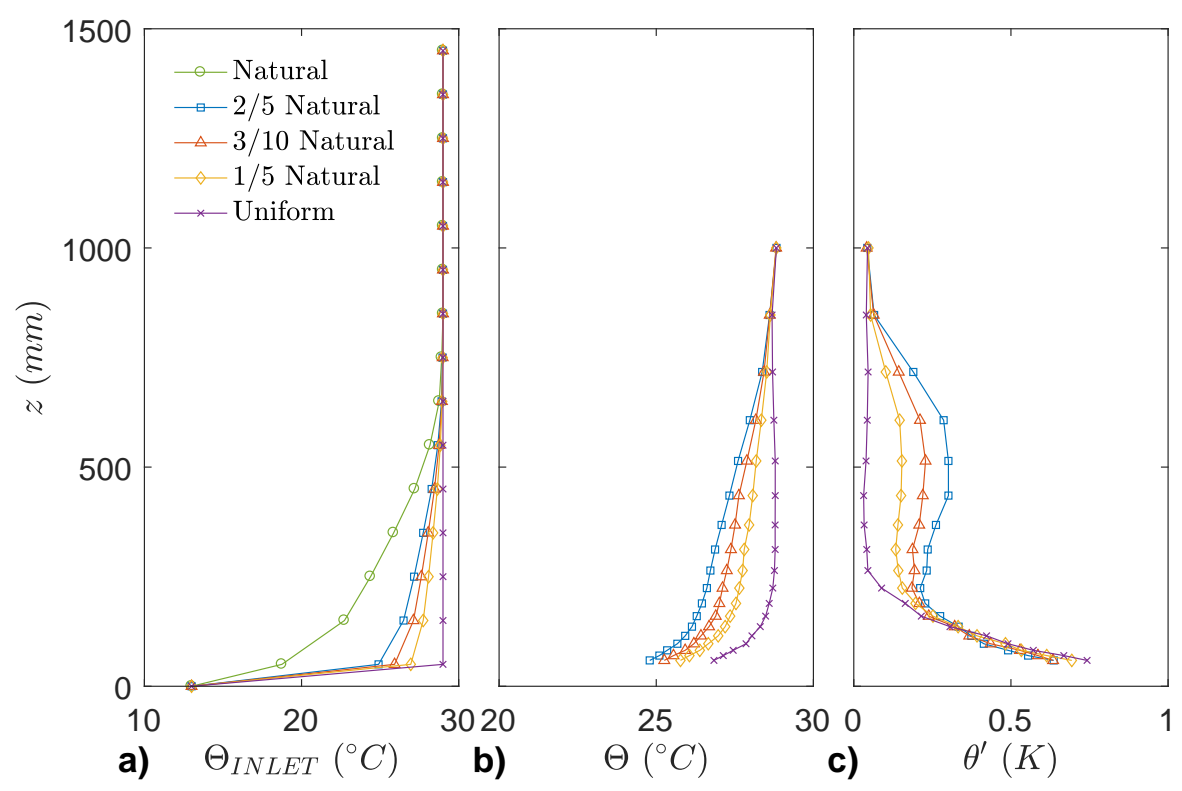

Figure 3: Effect of variation of inlet temperature profile gradient (a) on mean (b) and fluctuating (c) temperature profiles at $x=6480 \mathrm{~mm} . U_{\mathrm{REF}}=1.50 \mathrm{~m} / \mathrm{s}$ and $\Delta \Theta_{\mathrm{MAX}}=16^{\circ} \mathrm{C}$

(2014) and the resulting BL presented decreasing temperature fluctuations with height $z$, followed by a rise in the middle region which was attributed by the authors to a too large gradient of mean temperature in the same region. More promising is the approach experimented by Hancock and Hayden (2018). The idea was to impose the measured profile in a naturally-growing SBL (where "naturally-growing" is referred to a BL created just by friction with the cooled floor, without any flow generator or roughness element) as inlet temperature profile, starting from an initial uniform profile. The acquired temperature profile was stretched to fit the desired $\Delta \Theta_{\mathrm{MAX}}$ and BL height $\delta$ and applied to the inlet section with flow generators and roughness elements in place again. The resulting profile is shown in Fig. $3 \mathrm{a}$ and hence referred to as "Natural".

However, a direct application of such an initial condition (not shown here) created an undesired large peak in the middle region of the temperature fluctuation graph. Therefore, the original gradient was reduced applying corrective 
factors until the best solution was found. The other cases in Fig. 3 represent respectively a reduction of a factor $2 / 5,3 / 10,1 / 5$ of the "natural" one; the uniform temperature case is also shown. They were acquired after just $1.5 \mathrm{~m}$ of floor cooling, and so still in the "developing region" of the flow. The most significant effect of varying the inlet gradient is on the temperature fluctuation; in fact, even though the temperature standard deviation profiles show the same trend in the bottom part (Fig. 3r) a peak is present in the middle region for the $2 / 5$ case (and it would be even worse approaching the "natural" gradient). The peak is quite reduced for the $3 / 10$ case and disappears for the $1 / 5$ and the uniform profile. However, as compromise, the 3/10-reduced version of the inlet temperature profile was chosen as upstream condition for the following experiments.

\subsection{Reproducibility of stratification at different Reynolds numbers}

The wind velocity is normally not a critical parameter when simulating NBLs: with only a lower value limitation dictated by the requirement of Reynolds number independence, the range of usable velocities is mostly limited by the wind tunnel capabilities. On the other hand, when simulating thermally stratified BLs any small change in velocity produces a large effect on the stratification, being $R i_{b} \propto U^{-2}$. Lower velocity values become desirable, in particular, when larger $R i_{b}$ are requested, but the temperature difference parameter must be kept within a certain limit.

The purpose here is to investigate the similitude of artificially thickened SBLs obtained from different velocities but matching the same $R i_{b}$ by means of adjusting $\Delta \Theta_{\text {MAX }}$ accordingly. The reference velocity $\left(U_{\mathrm{REF}}\right)$ was set to 1.0 , 1.25 and $1.5 \mathrm{~m} / \mathrm{s}$, correspondent to $R e_{\delta}=U_{\delta} \delta / \nu$ (with $\nu$ air kinematic viscosity at $\left.\Theta_{0}\right)$ from $6.1 \times 10^{4}$ to $9.2 \times 10^{4}$. The stratification level was kept nominally constant by adjusting the $\Delta \Theta_{\text {MAX }}$ at the inlet (as shown in Table 1 in order to match a $R i_{b}$ of 0.14 in all three cases.

Fig. 4 shows the non-dimensional vertical profiles of mean streamwise velocity, Reynolds shear stress, mean temperature, vertical kinematic heat flux, 
Table 1: Main scale parameters for $R i_{b}=0.14$ case obtained with different $R e_{\delta}$

\begin{tabular}{lccc}
$\operatorname{Re}_{\delta}\left(\times 10^{4}\right)$ & 9.2 & 7.7 & 6.1 \\
\hline $\mathrm{U}_{\mathrm{REF}}(\mathrm{m} / \mathrm{s})$ & 1.50 & 1.25 & 1.00 \\
$\Delta \Theta_{\mathrm{MAX}}\left({ }^{\circ} \mathrm{C}\right)$ & 16.0 & 10.8 & 6.4 \\
$\mathrm{u}_{*} / \mathrm{U}_{\mathrm{REF}}$ & 0.053 & 0.053 & 0.053 \\
$\mathrm{z}_{0}(\mathrm{~mm})$ & 2.4 & 2.3 & 2.3 \\
$\mathrm{z}_{\mathrm{Oh}}(\mathrm{mm})$ & 0.021 & 0.007 & 0.013 \\
$\theta_{*}(\mathrm{~K})$ & 0.35 & 0.24 & 0.16 \\
$\delta / \mathrm{L}$ & 0.64 & 0.63 & 0.65 \\
$\mathrm{Ri}_{\mathrm{b}}$ & 0.14 & 0.14 & 0.14 \\
\hline
\end{tabular}

gradient Richardson number, Brunt-Väisälä frequency (calculated as $N_{B V}=$ $\sqrt{(g / \Theta)(\partial \Theta / \partial z)})$, streamwise and vertical velocity length scales. The latter were computed from the numerical integration of the autocorrelation coefficient, assuming the Taylor's hypothesis of "frozen turbulence". All the graphs indicate a fairly good agreement among the profiles of the three BLs. Also the values of $\delta / L$ in Table 1 appear to scale very well (with $\delta \approx 850 \mathrm{~mm}$ for all three cases), confirming that the stratification remained unchanged. To be noted that, even though in Fig. 4 only one profile at $x=12.5 \mathrm{~m}$ for each case is shown, the values in the table were estimated from the average of profiles at three different locations (as explained in paragraph 2.3.

The aerodynamic and thermal roughness lengths were estimated with a nonlinear least-squares fitting of Eq. 8 and 9 with the profiles of mean velocity and temperature, respectively. The displacement height was set equal to zero (here and for all the cases analysed) as indicated also by Cheng and Castro (2002) for the same roughness. Letting it varying freely led in some cases to inconsistent values without an effective improvement of the fit. The obtained values, despite being the result of different approximations and dependent by the methodology chosen so far, are consistent each other, since they do not differ too much in the three BLs. The $R i$ profile for the three cases coincides quite well with Eq. 12 for the same height for which the fitting is verified between the log- 

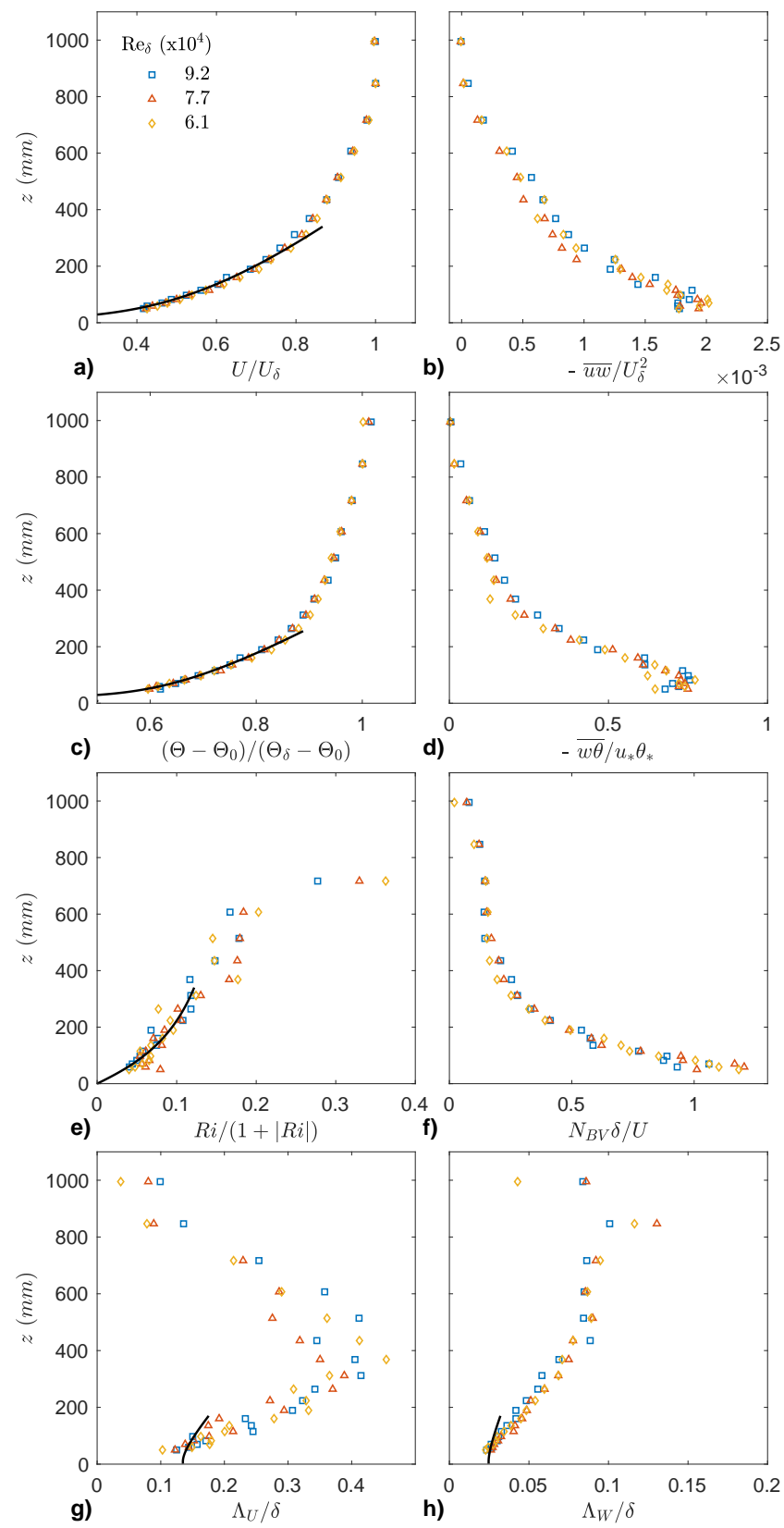

Figure 4: Profiles of mean streamwise velocity, Reynolds shear stress, mean temperature, vertical kinematic heat flux, gradient Richardson number, Brunt-Väisälä frequency, streamwise and vertical velocity integral lengthscales for different Reynolds numbers but same bulk Richardson number $\left(R i_{b}=0.14\right)$. Black lines are, respectively, (a) Eq. 8 . (c) Eq. 9 (e) Eq. 12 while (g) and (h) are from Kaimal (1973) 


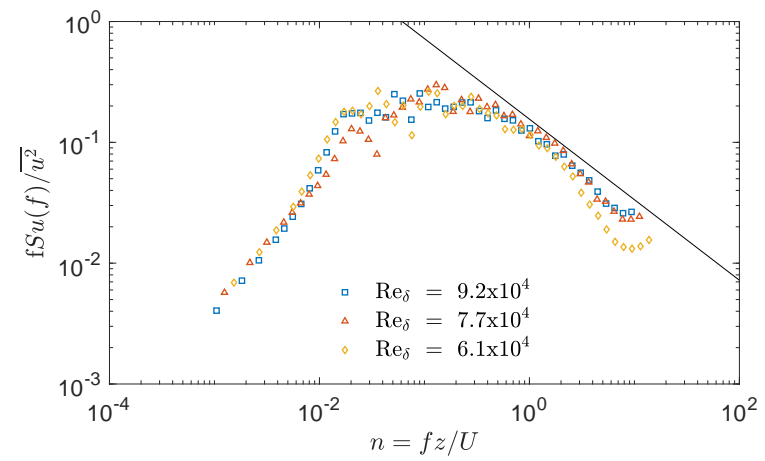

Figure 5: Power spectral density of streamwise velocity for different Reynolds numbers but same bulk Richardson number $\left(R i_{b}=0.14\right) . z=190 \mathrm{~mm}$. Black line is $-2 / 3$ slope reference.

law and the velocity profile. For the integral length scales in Fig. $4 \mathrm{~s}$ and $\mathrm{h}$ a comparison with field data is provided as well, by means of the empirical relations $\Lambda_{U}=0.082 z / R i$ and $\Lambda_{W}=0.015 z / R i$ from Kaimal (1973) (in which $R i$ was calculated using Eq. 12 .

Finally, Fig. 5 shows the power spectral density graph of streamwise velocity for the three cases considered here against the non-dimensional frequency $n=$ $f z / U$ (where $f$ is the dimensional one). The maximum peak frequency and the general shape is similar for all cases, while the slower case exhibits a steeper reduction at the higher frequencies of the inertial sub-range, also compared to the $-2 / 3$ reference line. This behaviour is expected and compatible with a reduction of the Reynolds number. Difference that, however, does not preclude the similitude of mean and turbulent quantities already commented.

The conclusion drawn from this data is the confirmation that for an artificially thickened BL, the same level of stability (in terms of ratio $\delta / L$ and similitude of non-dimensional mean and turbulent profiles) can be obtained for different velocities by matching the $R i_{b}$. Moreover, the reduction in $R e$ does not seem to affect mean and turbulent quantities. This result is also supported by the fact that the roughness Reynolds number $R e_{*}=u_{*} z_{0} / \nu$ (used to evaluate whether the surface is fully rough) is for the slowest case still greater than 8 , and thus larger than the minimum limit of 1 indicated by Snyder and Castro 
(2002) for sharp-edged roughness elements in a NBL.

\subsection{Comparison with NBL, different stratifications and surface roughness}

In this section a SBL characterised by $R i_{b}=0.21$ (obtained imposing $\Delta \Theta_{\mathrm{MAX}}$ $=16^{\circ} \mathrm{C}$ and $U_{\mathrm{REF}}=1.25 \mathrm{~m} / \mathrm{s}$ ) is compared with a NBL developing from the same spires.

Fig. 6 shows the stable (S) profiles at three streamwise locations compared with a neutral $(\mathrm{N})$ one, for which (for clarity) only the average profile at the same three locations is plotted. For the latter, the measured points differ from the average profile less than $\pm 0.5 \%$ on $U$ mean and less than $\pm 5 \%$ for the other quantities shown (except for the vertical length scale which differs up to 18\%).

In Fig. 6 the mean velocity reaches a maximum and the Reynolds shear stress profile approaches zero for approximately $z=850 \mathrm{~mm}$, which suggests a $\delta$ value of the same amount (equivalent in full-scale to a BL $170 \mathrm{~m}$ deep). Such a value is equal for stable and neutral stratifications. This allows to speculate that the combination of chosen spires and inlet temperature profile overcomes the effect of stability to reduce $\delta$. Also the general shape of the mean velocity profile is fairly similar between the two stratifications, but with the SBL characterised, as expected, by lower velocities at the bottom and higher at the top compared to the NBL. The aerodynamic roughness length is also almost the same between the two cases $\left(z_{0}=2.3 \mathrm{~mm}\right.$ for SBL and 2.2 for NBL). The small effect of the stratification on the mean velocity profile leads to the conclusion that such profile is mostly controlled by the spires (as also observed in Hancock and Hayden, 2018).

The reduction of turbulence due to stratification involves the entire BL and it is evident for the variances and the covariance of the velocity which appear up to $50 \%$ smaller. Same reduction is also experienced by the integral length scales, while the $u_{*}$ is about $30 \%$ lower (from $u_{*} / U_{\mathrm{REF}}=0.065$ to 0.047 ).

Fig. 7 presents a comparison between non-dimensional profiles of the two stable and one neutral BLs already introduced plus a more stable one obtained with the same $\Delta \Theta_{\mathrm{MAX}}=16^{\circ} \mathrm{C}$ but reducing the velocity to $U_{\mathrm{REF}}=1.0 \mathrm{~m} / \mathrm{s}$. 

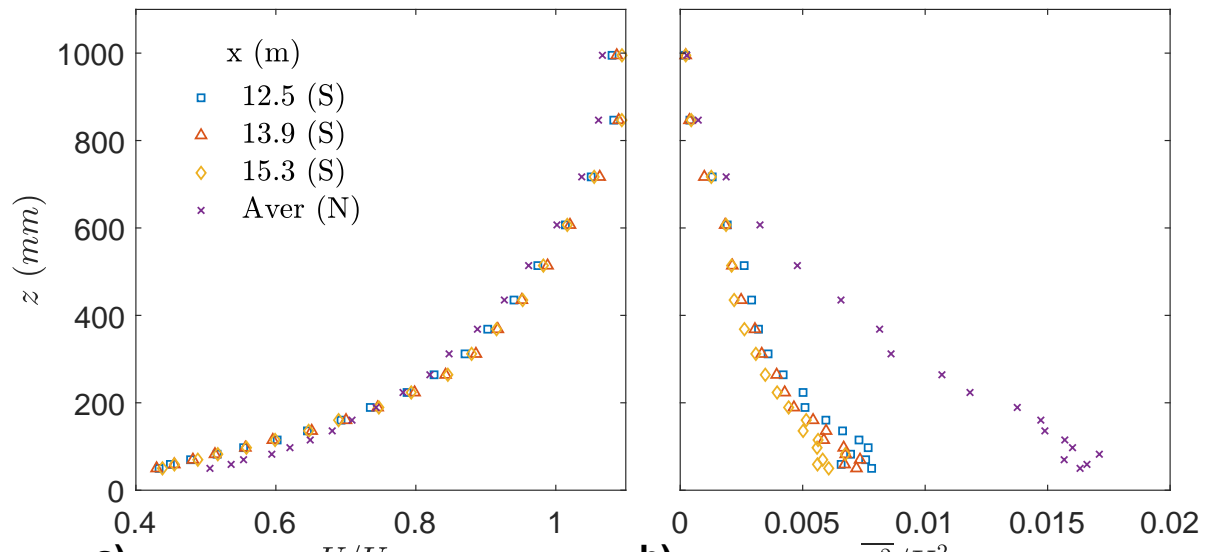

a) $U / U_{R E F}$

b)
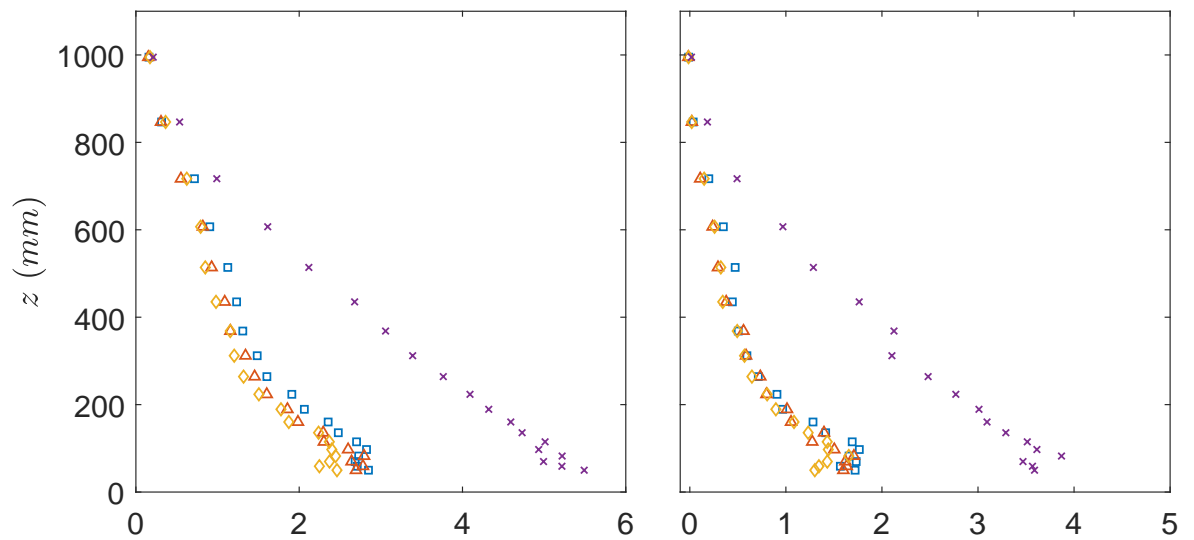

c) $\quad \overline{w^{2}} / U_{R E F}^{2} \quad \times 10^{-3} \mathbf{d}$ )
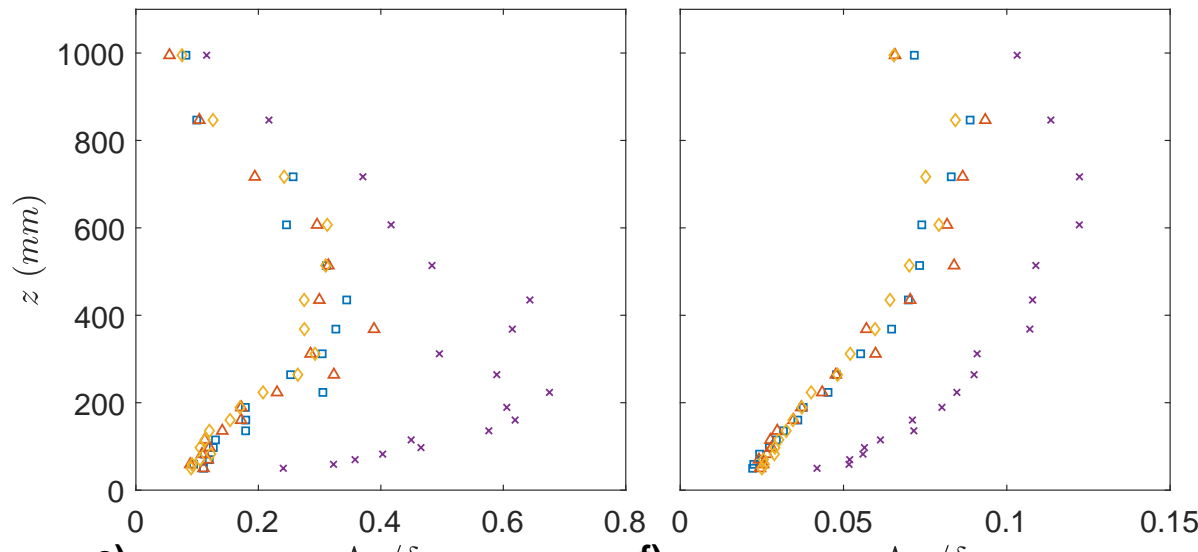

f)

$\Lambda_{W} / \delta$

Figure 6: Profiles of mean streamwise velocity, Reynolds shear stresses and integral length scales for a SBL with $R i_{b}=0.21$ and the reference NBL. The NBL profile is obtained as average of the ones acquired at the same three locations of the SBL. 

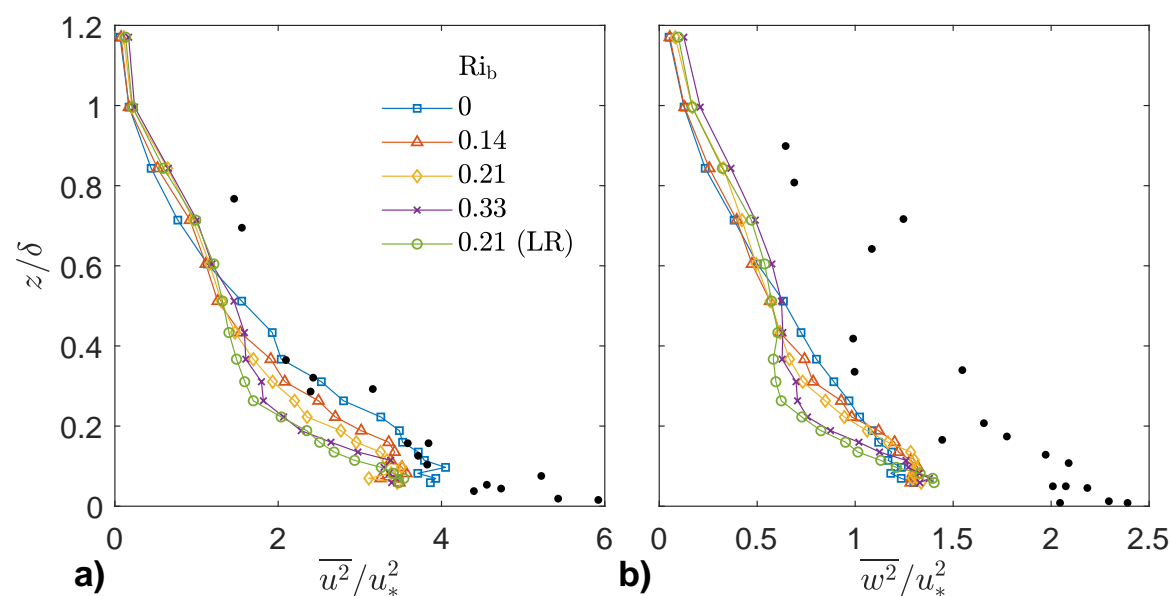

b)
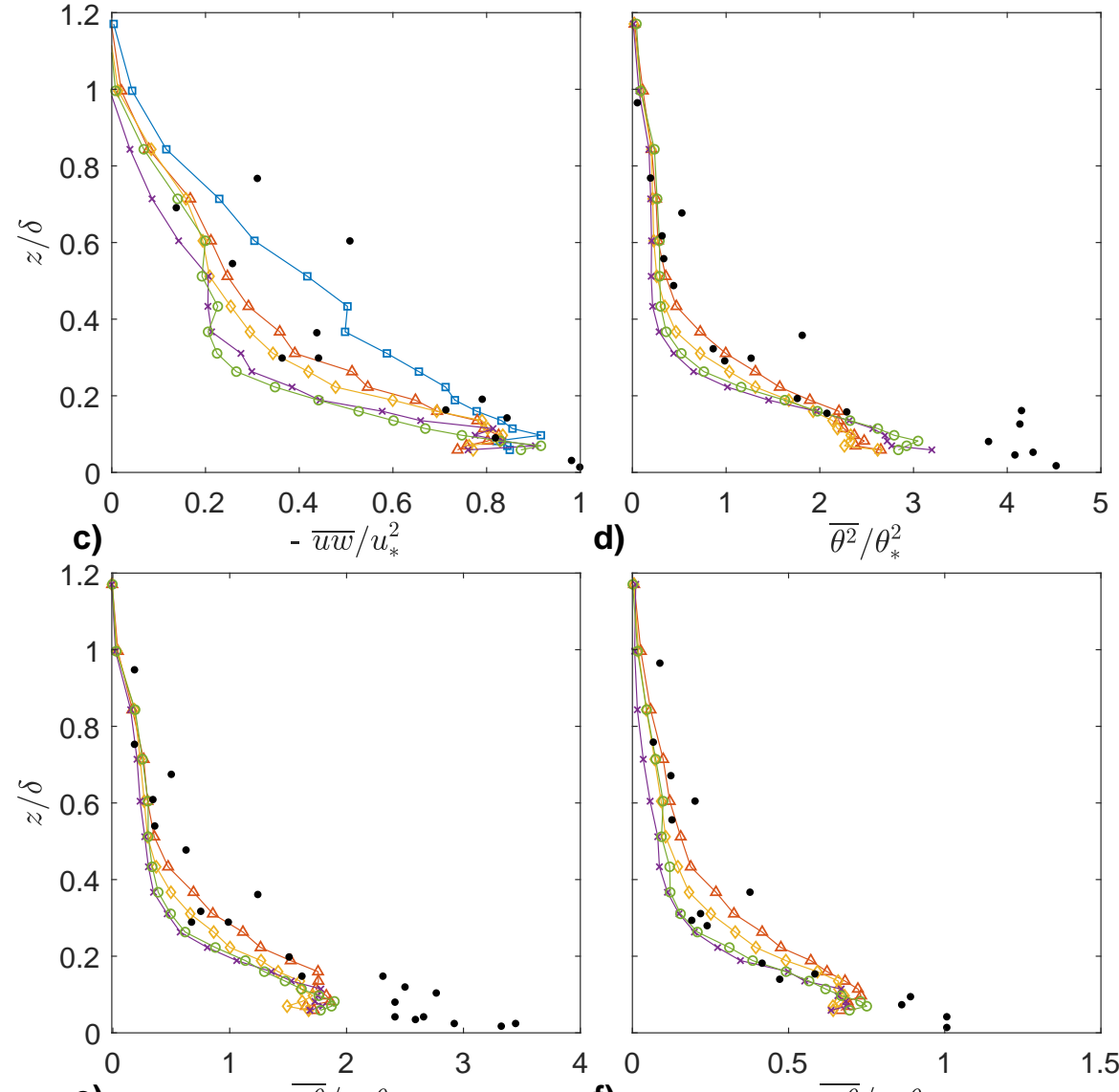

e) $\overline{u \theta} / u_{*} \theta_{*} \quad$ f)

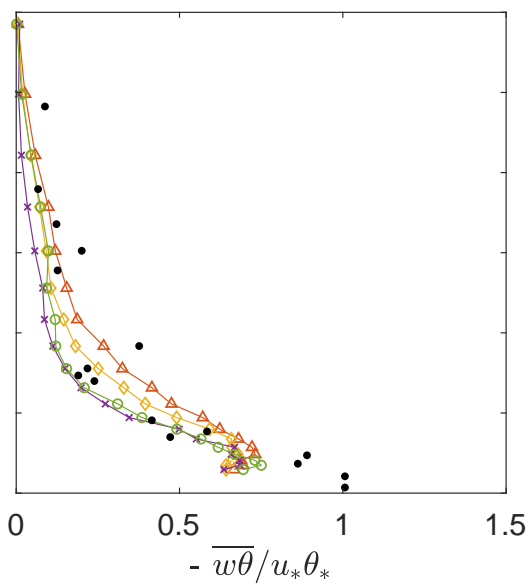

Figure 7: Profiles of non-dimensionalised streamwise and vertical velocity variance, Reynolds shear stress, temperature variance, streamwise and vertical kinematic heat flux for different level of stability and roughness. Black points are field data from Caughey et al. (1979) 
Table 2: Main scale parameters for reference neutral and three different stability cases obtained with different velocities, plus one lower roughness case

\begin{tabular}{lccccc}
\hline $\mathrm{Ri}_{\mathrm{b}}$ & 0 & 0.14 & 0.21 & 0.33 & $0.21(\mathrm{LR})$ \\
\hline $\mathrm{U}_{\mathrm{REF}}(\mathrm{m} / \mathrm{s})$ & 1.25 & 1.50 & 1.25 & 1.00 & 1.25 \\
$\Delta \Theta_{\mathrm{MAX}}\left({ }^{\circ} \mathrm{C}\right)$ & 0 & 16 & 16 & 16 & 16 \\
$\mathrm{u}_{*} / \mathrm{U}_{\mathrm{REF}}$ & 0.065 & 0.053 & 0.047 & 0.040 & 0.042 \\
$\mathrm{z}_{0}(\mathrm{~mm})$ & 2.2 & 2.4 & 2.3 & 2.4 & 0.6 \\
$\theta_{*}(\mathrm{~K})$ & - & 0.35 & 0.34 & 0.30 & 0.30 \\
$\delta / \mathrm{L}$ & 0 & 0.64 & 1.13 & 2.18 & 1.27 \\
\hline
\end{tabular}

A fifth case is also added, generated with the same settings of the third, but with a reduced roughness density (in the case the roughness elements rows were $720 \mathrm{~mm}$ apart) and characterised by a $z_{0}$ four times smaller. The main parameters of the five cases are summarised in Table 2. All the profiles shown are the result of averaging from three different locations (as for the NBL in Fig. 6). The longitudinal variability of the profiles ranges from a minimum of $\pm 2 \%$ for the temperature variance at $R i_{b} 0.14$ to $\pm 15 \%$ for the covariances when $R i_{b} 0.33$ (with the majority of the profiles below $10 \%$ ). In general, the variability increased with $R i_{b}$. Moreover, in the lower-roughness case this variability was found to be larger than the higher-roughness case with similar stability.

Among the three high-roughness stable cases, the largest differences in turbulent properties appear in the lower half of the BL. This behaviour is explainable by the fact that the cooling time is increased with a reduced velocity. This causes a larger reduction of turbulence in the bottom part, but the reduced vertical displacement due to the stratification prevents the modification farther away from the floor. This behaviour makes the Reynolds shear stress of the stable cases deviate from the almost-linear trend of the reference NBL. In the bottom half of the BL, the lower roughness case is much more similar to the $R i_{b}=0.33$ case with high roughness than to the high-roughness case with the same Richardson number $\left(R i_{b}=0.21\right)$. This trend is particularly evident in the $\overline{u w}$ graph. The results suggest that a reduction of the roughness has the 
same effect on the turbulence profiles of an increment of the stratification, at least in the bottom part of the BL. The upper part, on the contrary, seems to be less influenced by the roughness and the inlet conditions are predominant in shaping the profiles.

Despite the modifications described, all the profiles scale reasonably well with the surface shear stress. This is in contrast with what shown, e.g., by Ohya (2001), where for $R i_{b}>0.25$ the turbulence profiles were expected to collapse in the very stable regime. As mentioned in the introduction, Williams et al. (2017) indicated an even lower threshold $\left(R i_{b}>0.15\right)$ for the rough surface case. A possible explanation of this discrepancy is that the method employed to artificially thicken the BL, being originally developed for NBL, may not be suitable for very SBLs and further work would be necessary to come to a conclusion. On the other hand, Williams et al. (2017) also pointed out that the $R i_{b}$ could be a too crude indication to determine the transition in the stability regime. They quoted Flores and Riley (2011), claiming that the Reynolds number based on $L$ and $u_{*}\left(R e_{L}=L u_{*} / \nu\right)$ could possibly be a better indicator of the transition, found to happen for values of $R e_{L}$ lower than 100130 , independently from the surface roughness. For all the presented cases $R e_{L}$ was greater than 1000, so a very stable regime based on this criterion should not be expected. In Fig. 7 the field data measurements from Caughey et al. (1979) are reported as well. The agreement is reasonably good, in particular for the $R i_{b}=0.14$ case.

The final comments are on the lateral uniformity and standard error experienced with stable stratification and reference NBL. The former, looking at lateral profiles performed at a height of $300 \mathrm{~mm}$ spanning a length of $\pm 1000 \mathrm{~mm}$ from the centreline, was found to be generally quite good, with a mean velocity variation of the order of $\pm 2 \%$, less than 1 for the mean temperature, around $5 \%$ for the variances and $10 \%$ for the covariances. Similar values were obtained for the NBL.

The standard error on mean $U$ and $\Theta$ was less than $\pm 1 \%$, around $5 \%$ for the variances and $15 \%$ for the covariances. To be noted that the lateral (as well 
as the longitudinal) variability are of the order of the standard error. Similar quantities were found for the reference NBL. The standard error in the SBL was around $\pm 1 \%$ for mean velocity, $5 \%$ for the variances and $10 \%$ for the covariances. For the NBL, similar values were obtained, except the streamwise velocity variance for which the standard error was closer to $10 \%$.

\section{Convective boundary layer}

Similarly to the SBL, in order to simulate a CBL the temperature difference $\Delta \Theta_{\text {MAX }}$ and the flow velocity are the main ways to control the (unstable) stratification strength. The inlet temperature profile and the strength of the inversion layer imposed above the BL are other important parameters to consider, in particular as they where found to somewhat influence the lateral uniformity of the flow and temperature fields. Before considering the effect of this inlet temperature controls some considerations on the floor heater mats must be discussed. As already mentioned, the laboratory employs $2950 \mathrm{~mm}$ long rectangular heater panels, so that when placing them transversally on the floor, the last $275 \mathrm{~mm}$ on both sides are not heated (being the test section $3500 \mathrm{~mm}$ wide). In the past, Perspex panels were placed within the test section to reduce its width, but this remedy was not pursued in this case because the entire wind tunnel width was necessary for future experiments with the urban model. Therefore four different heater mats arrangements were considered, the first of which consisted in adjacent panels placed transversally, with $275 \mathrm{~mm}$ on both sides unheated. In Fig. 8 a lateral profile of temperature acquired with the double thermistor rake shows a reduction of up to $4 \%$ respect to the centre line $(2 \%$ in the region $\pm 1 \mathrm{~m})$. In the other configurations longitudinal panels were added on the sides in order to cover a wider region of the floor ${ }^{1}$. The graph shows that the configuration in which the largest part of the test section is heated does not present the best uni-

\footnotetext{
${ }^{1}$ In configuration 2 the longitudinal panel temperature was increased respect to the transversal one until the best uniformity was achieved. In Configurations 3 and 4 all the panels were set at the same temperature
} 
formity, with hot spots closer to the walls, while a reasonable compromise is the third configuration in which $110 \mathrm{~mm}$ are left unheated (without the complexity of adjusting the longitudinal panels temperature as for configuration 2).

\subsection{Temperature controls}

For the CBL an inlet temperature gradient and a capping inversion layer were considered separately. Differently from the SBL, using a NBL as starting point (uniform inlet temperature profile) and obtaining a CBL only by means of the heated floor was found acceptable. This approach was employed, for instance, by Fedorovich and Kaiser (1998) and Ohya and Uchida (2004). Differently, Hancock et al. (2013) suggested to adopt as inlet setting the temperature profile measured in a section downstream (starting from a uniform inlet profile) and iterating until a matching of the shape between the two was achieved. This method was tested in the present study with high-roughness conditions with the purpose of enhancing the longitudinal uniformity and reducing the fetch necessary to obtain a sufficiently developed CBL. However, the improvements were generally difficult to appreciate and hard to separate from the experimental scatter. Moreover, applying a negative inlet gradient was found to worsen the lateral uniformity (at least in the present case). Fig. 9 shows the lateral profiles of Reynolds shear stress, temperature variance and mean streamwise velocity for different inlet gradients. Three cases were considered: uniform temperature, "full gradient" from the direct application of the method and half gradient. The turbulence is less laterally uniform in the "full gradient" case in both graphs. While the Reynolds shear stress graph shows comparable results for the half gradient and uniform cases, the latter presents a slightly better uniformity in the central region of the temperature variance plot. Interestingly, the nonuniformity due to the gradient affects only turbulent quantities and heat fluxes, while mean velocity (Fig. 9d) and temperature profiles (not shown) seem not to be affected.

A capping inversion is a characteristic part of the CBL. Some previous studies (Ohya and Uchida, 2004, Fedorovich and Kaiser, 1998) paid great attention 

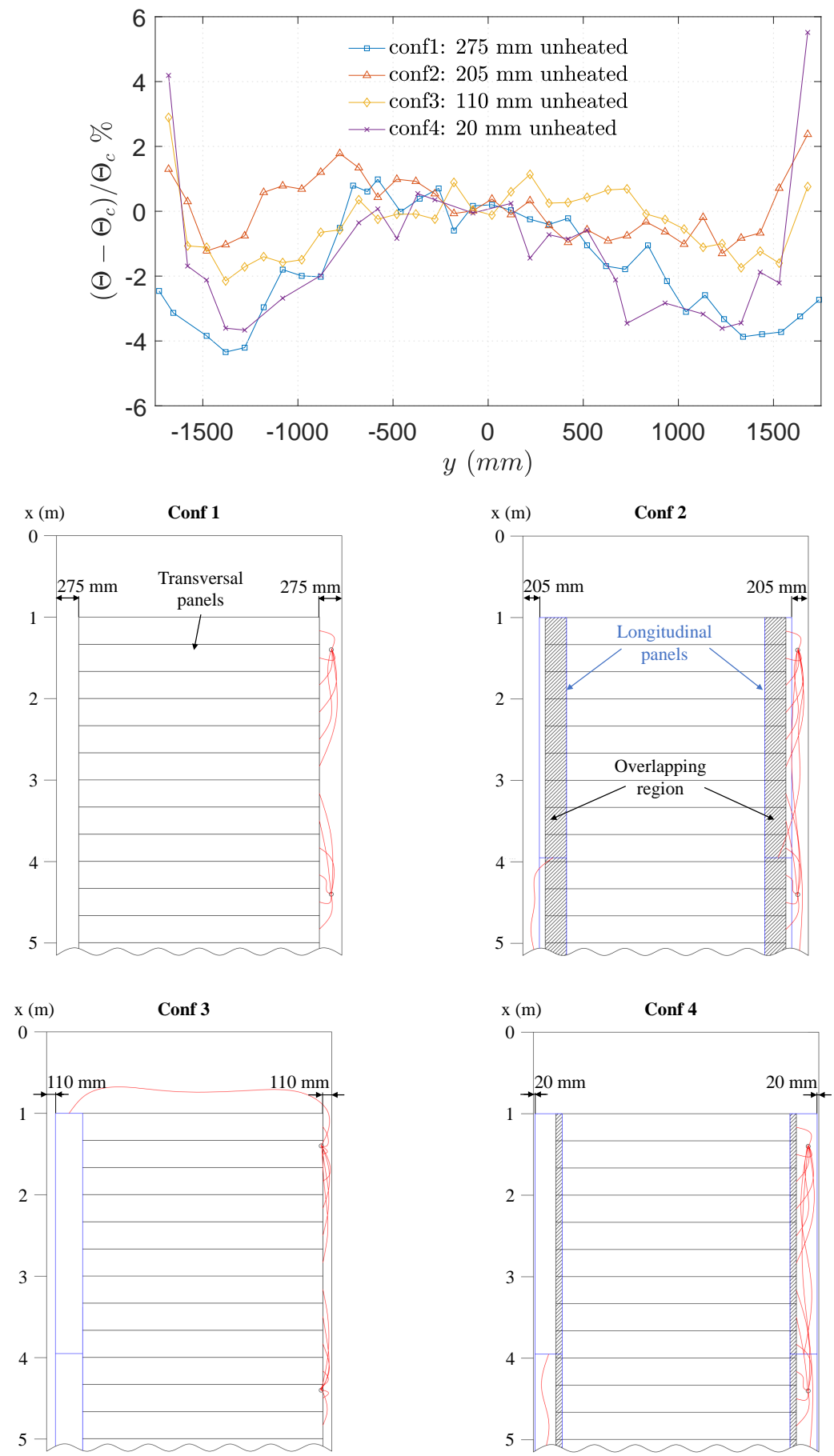

Figure 8: Comparison between different floor heater mats arrangements, whose plan-view is also represented $\left(x=14000 \mathrm{~mm}, z=300 \mathrm{~mm}, U_{\mathrm{REF}}=1.25 \mathrm{~m} / \mathrm{s}, \Delta \Theta_{\mathrm{MAX}} \approx 20^{\circ} \mathrm{C}\right) . \Theta_{c}$ is the temperature in the centreline 

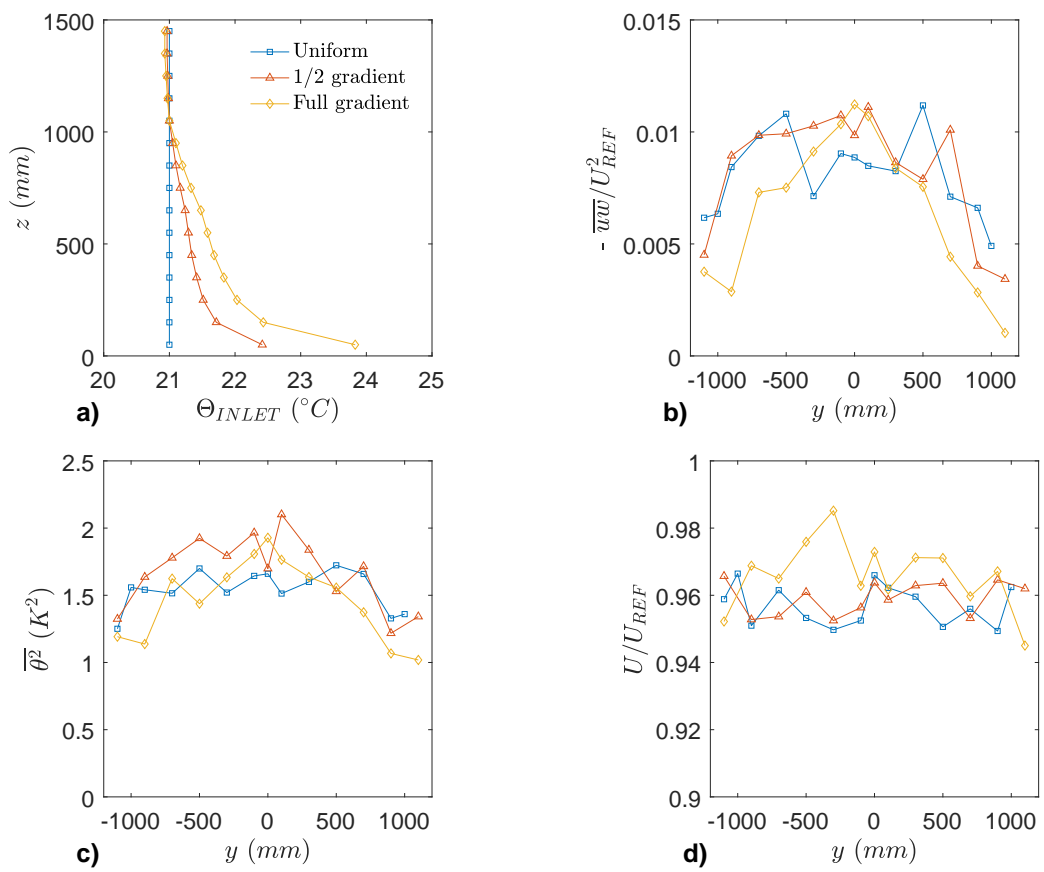

Figure 9: (a) Vertical profile of inlet temperature. Lateral profiles of Reynolds shear stress (b), temperature variance (c) and mean streamwise velocity (d) at $z=300 \mathrm{~mm}\left(U_{\mathrm{REF}}=1.0 \mathrm{~m} / \mathrm{s}\right.$, $\Theta_{0}=60^{\circ} \mathrm{C}, x=13900 \mathrm{~mm}$, floor configuration 4) 

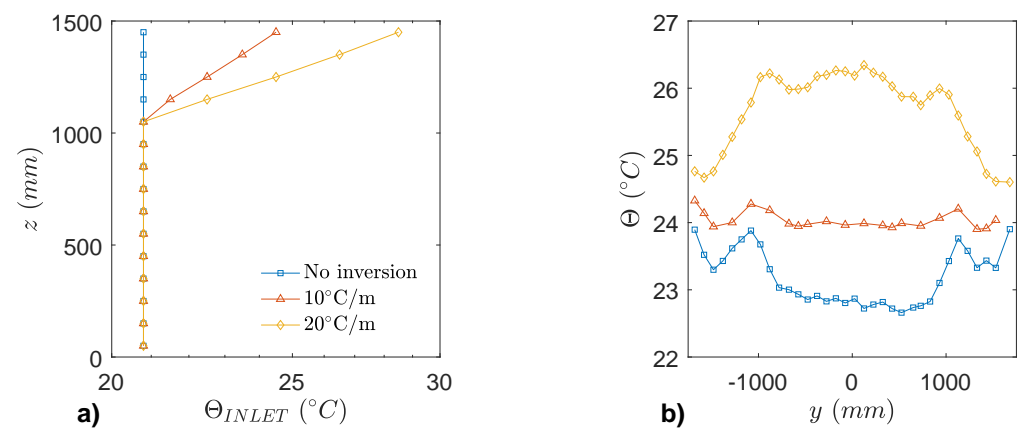

Figure 10: (a) Vertical profile of inlet temperature with different inversion strength. (b) Lateral profiles of temperature acquired with thermistor rake at $z=1225 \mathrm{~mm}$. $\left(U_{\mathrm{REF}}=\right.$ $\left.1.0 \mathrm{~m} / \mathrm{s}, \Theta_{0}=60^{\circ} \mathrm{C}, x=14500 \mathrm{~mm}\right)$

to the inversion layer and the entrainment. In the present study the focus is mainly on the simulation of the lower part of the CBL, which is most relevant for flow and dispersion studies in the urban environment. For this reason the correct representation of a capping inversion was not deemed essential. Weak linear inversions were applied above $1 \mathrm{~m}$ from the inlet and with a maximum gradient of $30^{\circ} \mathrm{C} / \mathrm{m}$. Inside this range no effects were experienced in the bottom half of the BL. However, a proper calibrated inversion capping the BL was found to greatly enhance the lateral uniformity. In Fig. 10 the lateral profile of temperature in the upper part with no inversion is compared with two cases with inversions (respectively with a 10 and $20^{\circ} \mathrm{C} / \mathrm{m}$ temperature increase). The lateral temperature profile appears colder in the central region compared to the sides. The opposite was found for the $20^{\circ} \mathrm{C} / \mathrm{m}$ inversion. On the other hand, employing the $10^{\circ} \mathrm{C} / \mathrm{m}$ inversion resulted in a better lateral uniformity of the temperature profiles. This fact seems to suggest that a proper inversion can be defined to match the temperature on the sides with the temperature in the central region. The beneficial effect of such an increased-temperature uniformity can be observed, for example, by comparing the vertical profiles of streamwise mean velocity in the centreline with the ones in the sides (Fig. 111).

The length of unheated floor after the inlet did not affect the CBL as it was noted in the SBL case. Only 1 and $4 \mathrm{~m}$ were tested and no significant 

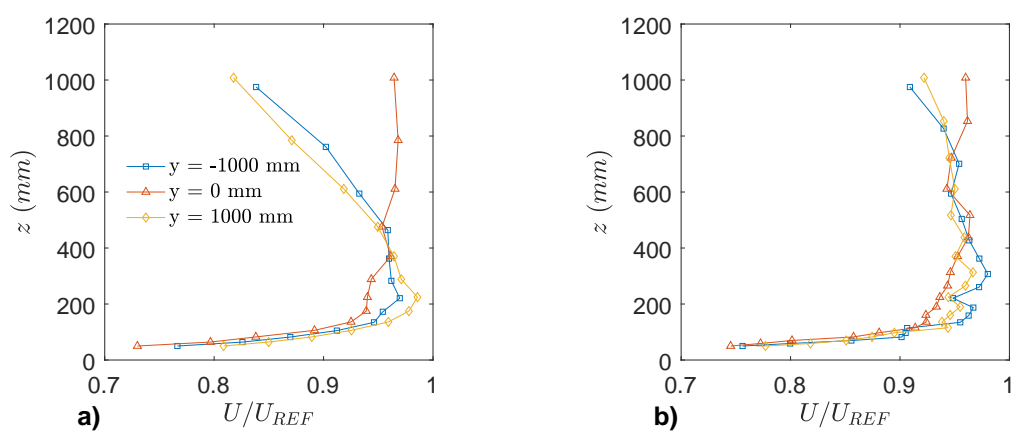

Figure 11: Mean velocity profiles with (a) no inversion and (b) inversion $10^{\circ} \mathrm{C} / \mathrm{m}$ at $x=$ $13900 \mathrm{~mm} .\left(U_{\mathrm{REF}}=1.0 \mathrm{~m} / \mathrm{s}, \Theta_{0}=60^{\circ} \mathrm{C}\right)$

improvements were observed by delaying the heating, apart from an undesired reduction of the level of instability. $1 \mathrm{~m}$ was the length used for all the results shown.

\subsection{Mean and turbulent profiles}

The strongest CBL case experimented here, obtained with uniform inlet temperature and an inversion of about $10^{\circ} \mathrm{C} / \mathrm{m}$ (adjusted to enhance the lateral uniformity), is presented in Fig. 12 and compared with a reference NBL. The longitudinal variability for the latter is comparable with the already presented NBL (obtained with the smaller spires). The main scale parameters are reported in Table 3

The streamwise mean velocity profile is greatly modified by the stratification: Eq. 10 readily fits with the bottom region, up to a sharp "knee" at $z \approx 150 \mathrm{~mm}$, while the region above shows constant velocity, compatible with the trend expected in a mixed-layer. The velocity profile presented in Fig. 2e, being a less unstable case $\left(R i_{b}=-0.5\right.$ instead of -1.5$)$, had a shape more similar to the NBL. The aerodynamic roughness length does not seem to be affected by the different stratification (being $2.0 \mathrm{~mm}$ for both $\mathrm{CBL}$ and $\mathrm{NBL}$ ), while $z_{0 h}$ has a value similar to the SBLs previously presented. The mean temperature also follows the similarity in the SL while the inversion appears notably reduced from the value imposed at the inlet, mostly due to mixing from below. Reynolds stresses have 

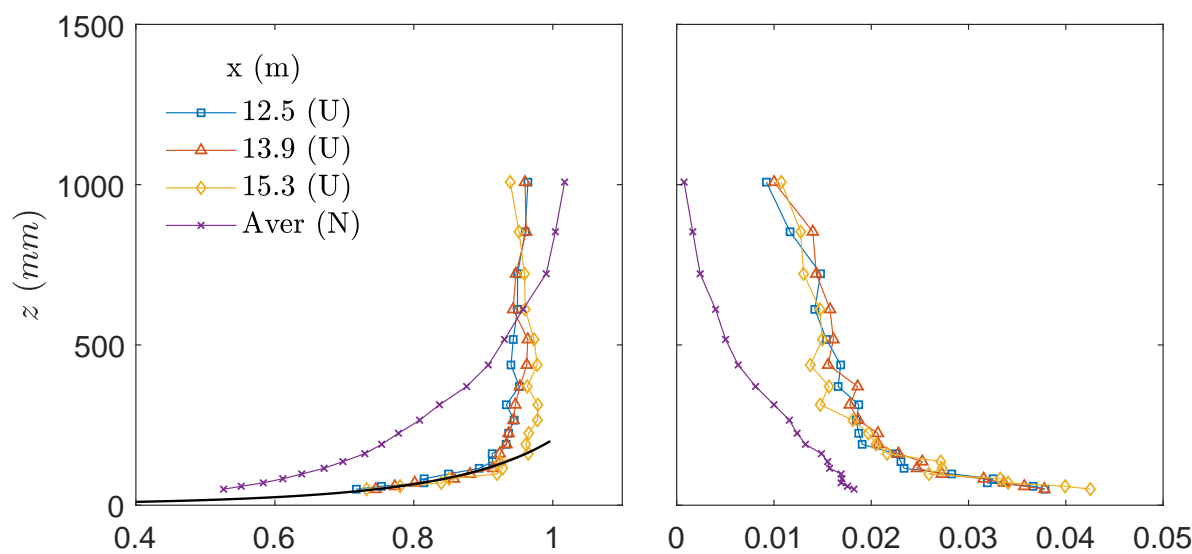

a)

$U / U_{R E F}$

b)
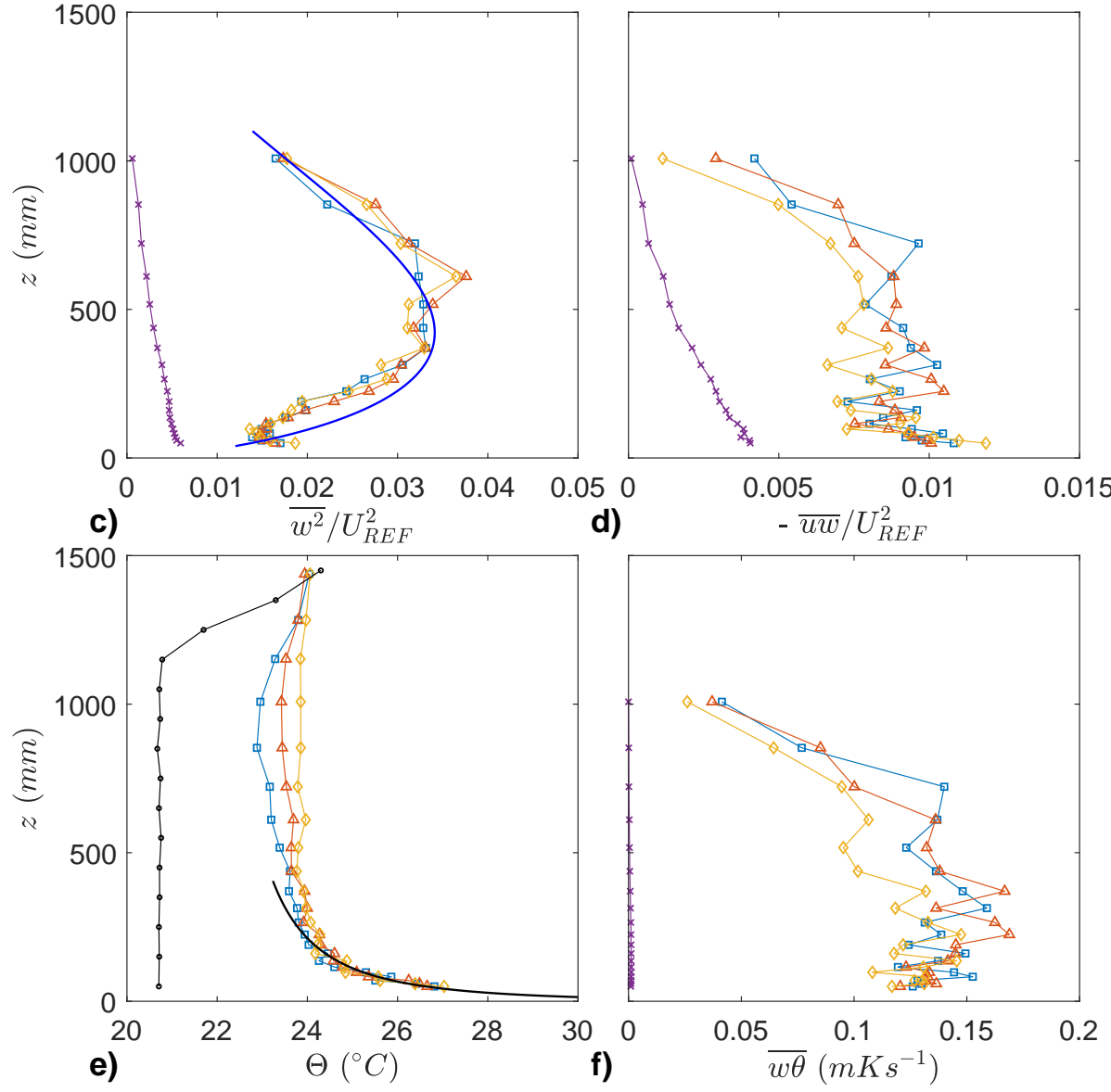

d) $\quad-\overline{u w} / U_{R E F}^{2}$

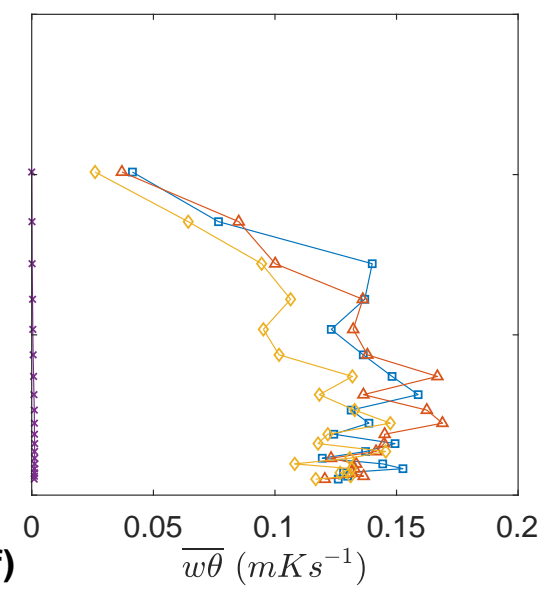

Figure 12: Profiles of mean velocity, Reynolds shear stresses, mean temperature and vertical kinematic heat flux for a CBL case $\left(R i_{b}=-1.5\right)$ and a reference neutral at the centreline. The NBL profile is obtained as average of the ones acquired at the same three locations of the CBL. Black lines in (a) and (e) are Eqs. 10. and 11 respectively; blue line in (c) is Eq. 15 black line with dots in (e) is the inlet temperature profile. 
Table 3: Main scale parameters for two different unstable cases obtained with different velocities and temperature settings, plus reference neutral. ( $\Delta \Theta_{\mathrm{MAX}}$ is indicated without considering the temperature inversion)

\begin{tabular}{lccc}
\hline & $\mathrm{N}$ & $\mathrm{HR} 2$ & $\mathrm{HR} 1(\mathrm{U})$ \\
\hline $\mathrm{U}_{\mathrm{REF}}(\mathrm{m} / \mathrm{s})$ & 2.0 & 1.25 & 1.0 \\
$\Delta \Theta_{\mathrm{MAX}}\left({ }^{\circ} \mathrm{C}\right)$ & 0 & -23 & -36 \\
$\delta(\mathrm{m})$ & 1.0 & 1.2 & 1.3 \\
$\mathrm{u}_{*} / \mathrm{U}_{\mathrm{REF}}$ & 0.065 & 0.084 & 0.100 \\
$\mathrm{z}_{0}(\mathrm{~mm})$ & 2.0 & 1.8 & 2.0 \\
$\theta_{*}(\mathrm{~K})$ & - & -0.78 & -1.4 \\
$\tilde{\theta}_{*}(\mathrm{~K})$ & - & 0.56 & 0.79 \\
$\mathrm{u}_{*} / \mathrm{w} *$ & - & 0.72 & 0.56 \\
$\delta / \mathrm{L}$ & 0 & -1.1 & -2.2 \\
$\mathrm{Ri}_{\mathrm{b}}$ & 0 & -0.5 & -1.5 \\
$\operatorname{Re}_{\delta}\left(\mathrm{x} 10^{4}\right)$ & 13.3 & 8.7 & 6.9 \\
\hline
\end{tabular}

much larger values compared to the neutral case $\left(u_{*} / U_{\mathrm{REF}}\right.$ is here 0.10 against 0.067). $\overline{u w}$ and $\overline{w \theta}$ have a similar trend, as already found for the $R i_{b}=-0.5$ case in Fig. 2, but the region of constant flux values extend now higher. The shape of the $\overline{w^{2}}$ profile is significantly different, showing an expected rise with $z$ followed by a decrease, instead of a monotonic reduction. Canonical similarity functions (see, e.g., Kaimal and Finnigan, 1994) do not seem to apply for this case, characterised by a low value of $\delta /|L|(\approx 2.2)$. Moreover, the ratio between friction velocity and convection velocity scale $u_{*} / w_{*}$ is here equal to 0.55 and, as reviewed by Fedorovich et al. (2001), when such ratio is larger than $\approx 0.35$ longitudinal rolls due to shear start to form, causing turbulence statistics to deviate from the free-convection case. Hancock et al. (2013) proposed a modified version to take into account the effect of the shear also in the ML. Here their relation for $\overline{w^{2}}$ in the ML is reported.

$$
\overline{w_{*}^{2}}=6.63\left(1+0.8 \frac{\delta}{|L|}\right)^{2 / 3}\left(\frac{z}{\delta}\right)^{2 / 3}\left(1-0.8 \frac{z}{\delta}\right)^{2}
$$


Eq. 15, in particular, is used here to estimate the BL depth $\delta$ by fitting with the $\overline{w^{2}}$ profile (Fig. $12 \mathrm{p}$ ): the value of $1.3 \mathrm{~m}$ provides a reasonable fitting. To be noted that at the chosen geometric ratio of 1:200 such height would correspond to $260 \mathrm{~m}$ on full-scale, which is quite small compared to normal CBL depths. Such a limitation, dictated by the dimensions of the wind-tunnel, is not deemed critical in this case as the models have, normally, considerably smaller dimensions at the urban micro-scale of interest.

Fig. 13 shows the non-dimensional Reynolds stresses, temperature fluctuation and vertical heat flux. The high-roughness case previously presented (here called HR1) is compared with the one characterised by the same roughness but weaker instability (HR2). The longitudinal variability for the plotted quantities was below $\pm 10 \%$, and for the variance of the velocity components for case HR1 was less than $\pm 2 \%$, four times lower than case HR2, suggesting that the increment in the mixing given by a larger $R i_{b}$ is beneficial for the longitudinal uniformity. The case U5 from Hancock et al. (2013) is also plotted and allows a comparison with a low-roughness case with similar instability (in this run $h / L \approx 1.26$ and $\left.u_{*} / U_{\mathrm{REF}}=0.055\right)$. The case E2 from Ohya and Uchida (2004) is reported as well (characterised by $h / L=3.11$ ). The $\overline{u^{2}}$ graph shows a good agreement between case HR2 and U5, with similar instability but different roughness. The same can be said for the $\overline{w^{2}}$ profiles. Again for $\overline{u^{2}} / w_{*}^{2}$ case HR1 presents lower values compared with the weaker case HR2. As far as nondimensional temperature variance and vertical heat flux are concerned, a good agreement is shown between all the presented experimental cases. The resemblance with the data from Ohya and Uchida (2004) is particularly significant, since no spires where used in their experiments.

Comparison with field measurements is provided by means of Caughey and Palmer (1979), Wilczak and Phillips (1986) and Wood et al. (2010). The nondimensional streamwise velocity variance experiences a degree of variability among the different authors comparable with the one found experimentally. For the vertical velocity variance the best agreement is found with Wilczak and Phillips (1986), while Caughey and Palmer (1979) and Wood et al. (2010) report 

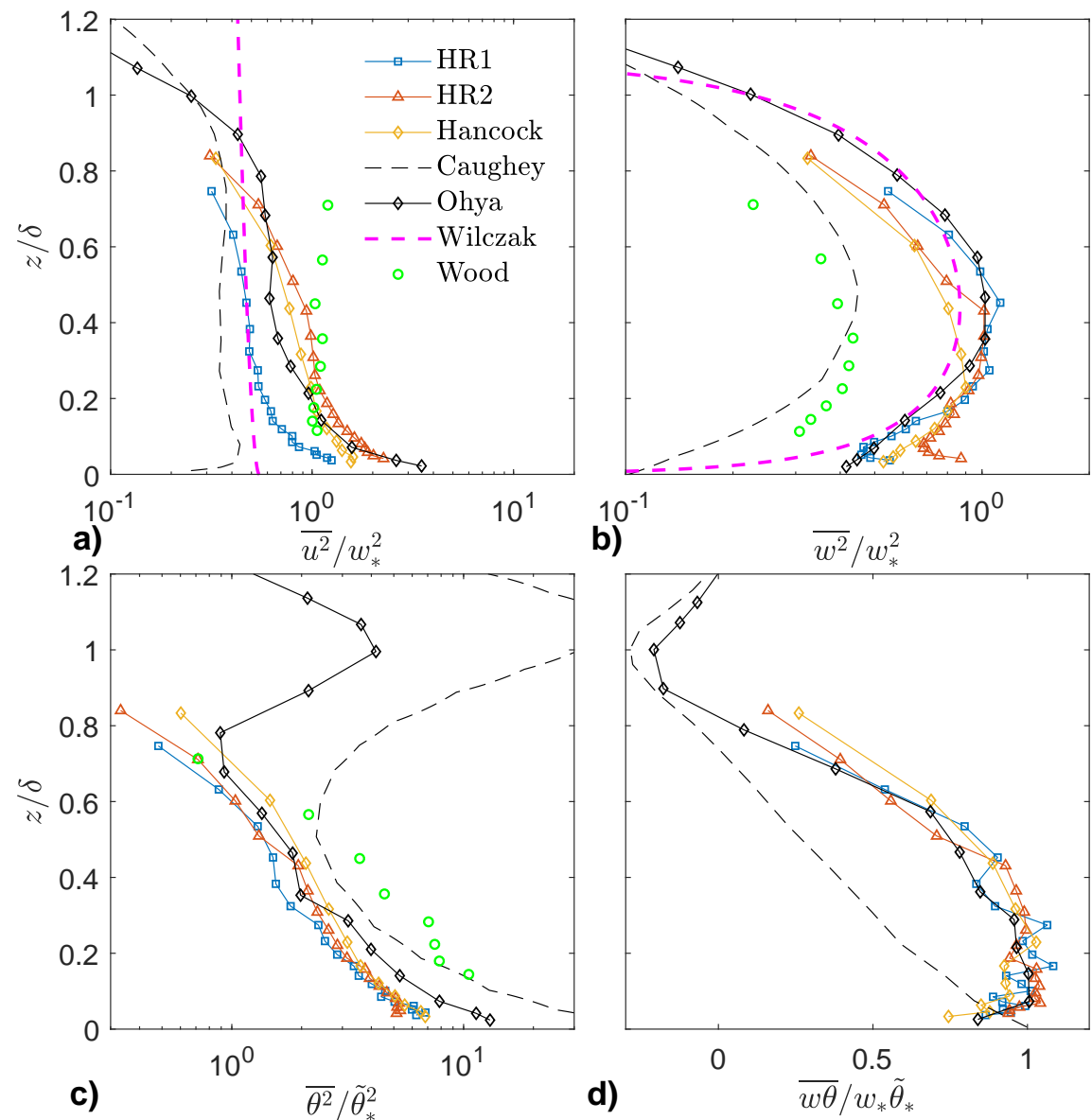

b)

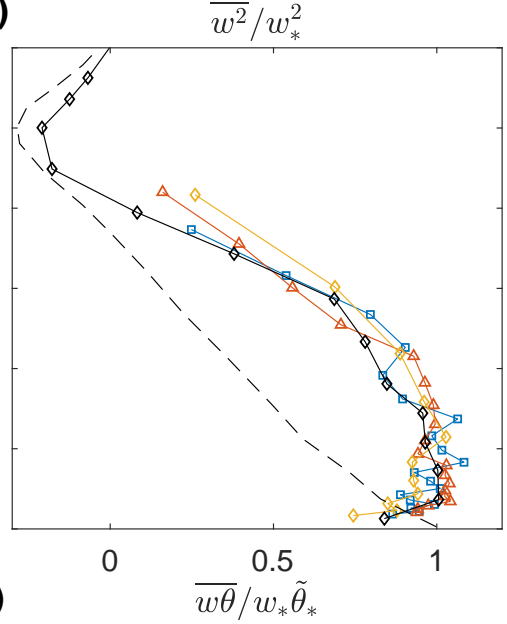

Figure 13: Profiles of non-dimensional Reynolds stresses, temperature variance and vertical kinematic heat flux. Data is compared with Hancock et al. (2013) (case U5), Caughey and Palmer (1979), Ohya and Uchida (2004) (case E2), Wilczak and Phillips (1986) and Wood et al. (2010) 
lower values (the opposite for the temperature variance). Finally, the vertical heat flux differs from the canonical linear trend found in field data. However, the result is close to what found by Ohya and Uchida (2004) for similar instability. To be noted that for stronger instability cases Ohya and Uchida (2004) obtained profiles of $\overline{w \theta}$ closer to the canonical linear.

As for the SBL the final comment is on the lateral uniformity and standard error. The spanwise variation of mean quantities was about $\pm 1 \%$, around $10 \%$ for the variances and 15 for the covariances. For the reference NBL the turbulence lateral variation was smaller, around $6 \%$ for the variance and 10 for $\overline{u w}$. The standard error on mean $U$ and $\Theta$ was about $\pm 1 \%$, up to $10 \%$ for the variances and up to $18 \%$ for the covariances. Finally, the reference NBL experienced values of variability and standard error of about $5 \%$ for variances and up to $18 \%$ for $\overline{u w}$.

\section{Conclusion}

Experiments involving the simulation of stable and convective boundary layers over a very rough surface have been performed in a thermally-stratified wind tunnel. Artificial thickening by means of Irwin's spires was used to accelerate the formation of a sufficiently deep boundary layer, suitable for urban-like boundary layer flow and dispersion studies.

For the stable boundary layer, the methodology presented in Hancock and Hayden (2018) for low-roughness offshore surface conditions has been successfully applied to cases with higher-roughness. The reproducibility of mean and turbulent profiles at different Reynolds numbers by matching the bulk Richardson number has been verified. Different levels of stratification produced modifications in the turbulence profiles of the lower half of the BL, but little or no change in the region above. The same can be said for the effect of the surface roughness, whose reduction was found to produce results similar to what observed after an increase in the stratification. A case with stronger stability 
(in terms of bulk Richardson number greater than 0.25 ) was simulated but the turbulence profiles continued to scale with the lower stability cases, suggesting that the employed spires may not be suitable to simulate such extreme condition, though further studies are needed. The results were in reasonably good agreement with field measurements (Caughey et al., 1979).

For the simulation of a convective boundary layer, great attention was given to the flow uniformity inside the test section. The selection of a non-uniform inlet temperature profile was in this case found not as determinant as for the stable boundary layer to improve the longitudinal uniformity, while the application of a calibrated capping inversion considerably improved the lateral uniformity. The non-dimensional vertical profiles of turbulent quantities and heat fluxes, did not seem to be influenced by roughness (by a comparison with Hancock et al., 2013), suggesting, again, that changes of roughness produce only local effects in the generated boundary layer. Good agreement is also shown with Ohya and Uchida (2004), in which no spires were employed.

\section{Acknowledgments}

This work was funded by the EPSRC (grant EP/P000029/1) and by the Department of Mechanical Engineering Sciences (University of Surrey). The authors confirm that all wind tunnel data are fully available without restriction from https://doi.org/10.6084/m9.figshare.5993572.

\section{References}

Argyle, P., Watson, S., 2012. A study of the surface layer atmospheric stability at two UK offshore sites, in: European wind energy association conference, pp. 16-19.

Arya, S.P.S., Plate, E.J., 1969. Modeling of the Stably Stratified Atmospheric Boundary Layer. doi:10.1175/1520-0469(1969) 26<656:MOTSSA>2 . 0.C0;2 
Caughey, S.J., Palmer, S.G., 1979. Some aspects of turbulence structure through the depth of the convective boundary layer. Quarterly Journal of the Royal Meteorological Society 105, 811-827. doi 10.1002/qj.49710544606.

Caughey, S.J., Wyngaard, J.C., Kaimal, J.C., 1979. Turbulence in the evolvn ing stable boundary layer. J. Atmos. Sci. 36, 1041-1052. doi 10.1175/ 1520-0469(1979)036<1041:TITESB>2.0.CO;2.

Cheng, H., Castro, I., 2002. Near wall flow development after a step change in surface roughness. Boundary-layer Meteorology 105, 411-432. doi 10.1023/ A:1020355306788.

Cheng, H., Hayden, P., Robins, A.G., Castro, I.P., 2007. Flow over cube arrays of different packing densities. Journal of Wind Engineering and Industrial Aerodynamics 95, 715-740. doi $10.1016 / \mathrm{j}$. jweia.2007.01.004.

Counihan, J., 1969. An improved method of simulating an atmospheric boundary layer in a wind tunnel. Atmospheric Environment 3, 197-214. doi $10.1016 / 0004-6981(69) 90008-0$.

Fedorovich, E., 2004. Dispersion of passive tracer in the atmospheric convective boundary layer with wind shears: A review of laboratory and numerical model studies. Meteorology and Atmospheric Physics 87, 3-21. doi:10.1007/s00703-003-0058-3.

Fedorovich, E., Kaiser, R., 1998. Wind tunnel model study of turbulence regime in the atmospheric convective boundary layer, in: Buoyant convection in geophysical flows. Springer Netherlands, pp. 327-370. doi 10.1007/ 978-94-011-5058-3.

Fedorovich, E., Nieuwstadt, F.T.M., Kaiser, R., 2001. Numerical and Laboratory Study of Horizontally Evolving Convective Boundary Layer. Part II: Effects of Elevated Wind Shear and Surface Roughness. Journal of the Atmospheric Sciences 58, 546-560. doi 10.1175/1520-0469(2001)058<0546: NALSOH $>2.0 . \mathrm{CO} ; 2$. 
Fedorovich, E., Rau, M., Kaiser, R., Plate, E., 1996. Wind Tunnel Study of Turbulent Flow Structure in the Convective Boundary Layer Capped by a 7 Temperature Inversion. doi $10.1175 / 1520-0469$ (1996) 053<1273:WTSOTF $>$ $2.0 . \mathrm{CO} ; 2$

Flores, O., Riley, J.J., 2011. Analysis of Turbulence Collapse in the Stably Stratified Surface Layer Using Direct Numerical Simulation. Boundary-Layer Meteorology 139, 241-259. doi:10.1175/1520-0469(1969) 26<656:MOTSSA $>$ $2.0 . \mathrm{CO} ; 2$

Hancock, P.E., Hayden, P., 2016. Wind-tunnel simulation of stably stratified atmospheric boundary layers. Journal of Physics: Conference Series 753, 032012. doi $10.1088 / 1742-6596 / 753 / 3 / 032012$.

Hancock, P.E., Hayden, P., 2018. Wind-Tunnel Simulation of Weakly and Moderately Stable Atmospheric Boundary Layers. Boundary-Layer Meteorology , 1-29doi $10.1007 /$ s10546-018-0337-7

Hancock, P.E., Pascheke, F., 2014. Wind-Tunnel Simulation of the Wake of a Large Wind Turbine in a Stable Boundary Layer. Part 1: The Boundaryn Layer Simulation. Boundary-Layer Meteorology 151, 3-21. doi 10.1007/ s10546-013-9886-y.

Hancock, P.E., Zhang, S., Hayden, P., 2013. A Wind-Tunnel Artificially-Thickened Simulated Weakly Unstable Atmospheric Boundary Layer. Boundary-Layer Meteorology 149, 355-380. doi 10.1007/ s10546-013-9847-5.

Heist, D.K., Castro, I.P., 1998. Combined laser-doppler and cold wire anemometry for turbulent heat flux measurement. Experiments in Fluids 24, 375-381. doi $10.1007 / \mathrm{s} 003480050186$.

Hibberd, M.F., Sawford, B.L., 1994. A Saline Laboratory Model of the Planetary Convective Boundary Layer. Boundary-Layer Meteorology 67, 229-250. doi:https://doi.org/10.1007/BF00713143. 
Högström, U., 1988. Non-Dimensional Wind and Temperature Profiles in the Atmospheric Surface Layer: A Re-Evaluation. Boundary-Layer Meteorology 42, 55-78. doi:10.1007/978-94-009-2935-7_6.

Irwin, H.P.A.H., 1981. The design of spires for wind simulation. Journal of Wind Engineering and Industrial Aerodynamics 7, 361-366. doi 10.1016/ 0167-6105(81) 90058-1.

Kaimal, J.C., 1973. Turbulence spectra, length scales and structure parameters in the stable surface layer. Boundary-Layer Meteorology 4, 289-309. doi 10 . 1007/BF02265239

Kaimal, J.C., Finnigan, J.J., 1994. Atmospheric boundary layer flows: their structure and measurement. volume 72. Oxford University Press. doi 10. 1016/0021-9169(95)90002-0.

Meroney, R.N., 1998. Wind tunnel simulation of convective boundary layer phenomena: simulation criteria and operating ranges of laboratory facilities., in: et al. Plate, E. (Ed.), Buoyant convection in geophysical flows. Kluwer, pp. 313-326. doi $10.1007 / 978-94-011-5058-3 \_14$.

Monin, A.S., Obukhov, A.M., 1954. Basic laws of turbulent mixing in the surface layer of the atmosphere. Contrib. Geophys. Inst. Acad. Sci. USSR 24, 163-187.

Ogawa, Y., Diosey, P.G., Uehara, K., Ueda, H., 1985. Wind tunnel observation of flow and diffusion under stable stratification. Atmospheric Environment (1967) 19, 65-74. doi 10.1016/0004-6981(85)90136-2

Ohya, Y., 2001. Wind-tunnel study of atmospheric stable boundary layers over a a rough surface. Boundary-Layer Meteorology 98, 57-82. doi:10.1023/A: 1018767829067 .

Ohya, Y., Neff, D.E., Meroney, R.N., 1997. Turbulence Structure in a Stratified Boundary Layer Under Stable Conditions. Boundary-Layer Meteorology 83, 139-162. doi:10.1023/A: 1000205523873 . 
Ohya, Y., Uchida, T., 2003. Turbulence structure of stable boundary layers with a near-linear temperature profile. Boundary-Layer Meteorology 108, 19-38. doi:10.1023/A:1023069316164

Ohya, Y., Uchida, T., 2004. Laboratory and numerical studies of the convective boundary layer capped by a strong inversion. Boundary-Layer Meteorology 112, 223-240. doi:10.1023/B:BOUN.0000027913.22130.73.

Oke, T.R., 1987. Boundary layer climates. 2nd ed., London. doi 10.1016/ 0012-8252(90)90005-G

Robins, A., Castro, I., Hayden, P., Steggel, N., Contini, D., Heist, D., John Taylor, T., 2001. A wind tunnel study of dense gas dispersion in a stable boundary layer over a rough surface. Atmospheric Environment 35, 22532263. doi:10.1016/S1352-2310(01)00073-5.

Snyder, W.H., Castro, I.P., 2002. The critical Reynolds number for rough-wall boundary layers. Journal of Wind Engineering and Industrial Aerodynamics 90, 41-54. doi:10.1016/S0167-6105(01)00114-3.

Stull, R.B., 1988. An Introduction to Boundary Layer Meteorology. volume 13. Springer Netherlands. doi 10.1007/978-94-009-3027-8.

Wilczak, J.M., Phillips, M.S., 1986. An indirect Estimation of Convective Boundary Layer Structure for Use in Pollution Dispersion models. Journal of climate and applied meteorology 25, 1609-1624. doi 10.1175/ 1520-0450(1986) 025<1609:AIEOCB>2.0.CO;2

Williams, O., Hohman, T., Van Buren, T., Bou-Zeid, E., Smits, A.J., 2017. The effect of stable thermal stratification on turbulent boundary layer statistics. Journal of Fluid Mechanics 812, 1039-1075. doi 10.1017/jfm.2016.781.

Willis, G.E., Deardorff, J.W., 1974. A Laboratory Model of the Unstable Planetary Boundary Layer. Journal of the Atmospheric Sciences 31, 1297-1307. doi:10.1175/1520-0469(1974)031<1297:ALMOTU>2.0.CO;2. 
${ }_{767}$ Wood, C.R., Lacser, A., Barlow, J.F., Padhra, A., Belcher, S.E., Nemitz, E., 768 Helfter, C., Famulari, D., Grimmond, C.S.B., 2010. Turbulent Flow at $190 \mathrm{~m}$ 769 Height Above London During 2006-2008: A Climatology and the Applicability 770 of Similarity Theory. Boundary-Layer Meteorology 137, 77-96. doi 10.1007/ $771 \quad$ S10546-010-9516-x. 